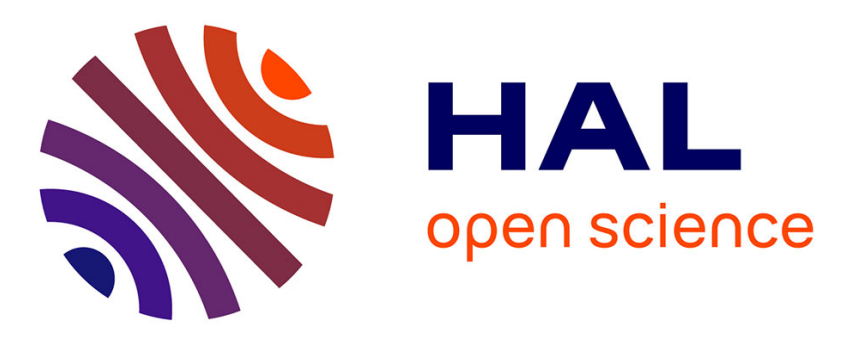

\title{
Investigation of advanced strain-path dependent material models for sheet metal forming simulations
}

\author{
Badis Haddag, Tudor Balan, Farid Abed-Meraim
}

\section{To cite this version:}

Badis Haddag, Tudor Balan, Farid Abed-Meraim. Investigation of advanced strain-path dependent material models for sheet metal forming simulations. International Journal of Plasticity, 2006, 23 (6), pp.951-979. 10.1016/j.ijplas.2006.10.004 . hal-00755503

\section{HAL Id: hal-00755503 https://hal.science/hal-00755503}

Submitted on 21 Nov 2012

HAL is a multi-disciplinary open access archive for the deposit and dissemination of scientific research documents, whether they are published or not. The documents may come from teaching and research institutions in France or abroad, or from public or private research centers.
L'archive ouverte pluridisciplinaire HAL, est destinée au dépôt et à la diffusion de documents scientifiques de niveau recherche, publiés ou non, émanant des établissements d'enseignement et de recherche français ou étrangers, des laboratoires publics ou privés. 


\title{
Investigation of advanced strain-path dependent material models for sheet metal forming simulations
}

\author{
Badis HADDAG, Tudor BALAN*, Farid ABED-MERAIM \\ Laboratoire de Physique et Mécanique des Matériaux, L.P.M.M., UMR CNRS 7554, \\ ENSAM de Metz, 4 rue Augustin Fresnel, 57078 Metz Cedex 03, France.
}

\begin{abstract}
Sheet metal forming processes often involve complex loading sequences. To improve the prediction of some undesirable phenomena, such as springback, physical behavior models should be considered. This paper investigates springback behavior predicted by advanced elastoplastic hardening models which combine isotropic and kinematic hardening and take strain-path changes into account. A dislocation-based microstructural hardening model formulated from physical observations and the more classical cyclic model of Chaboche have been considered in this work. Numerical implementation was carried out in the ABAQUS software using a return mapping algorithm with a combined backward Euler and semi-analytical integration scheme of the constitutive equations. The capability of each model to reproduce transient hardening phenomena at abrupt strain-path changes has been shown via simulations of sequential rheological tests. A springback analysis of strip drawing tests was performed in order to emphasize the impact of several influential parameters, namely: process, numerical, and behavior parameters. The effect of the two hardening models with respect to the process parameters has been specifically highlighted.
\end{abstract}

Keywords: Sheet metal forming; Isotropic-kinematic hardening; Strain-path change; Transient hardening; Implicit integration scheme; Finite element simulation; Springback predictions.

* Corresponding author. Address: ENSAM de Metz, 4 rue Augustin Fresnel, 57078 Metz Cedex 03, France. Tel.: +(33) 3.87.37.54.60; Fax: +(33) 3.87.37.54.70.

E-mail address: tudor.balan@metz.ensam.fr 


\section{Introduction}

The development of new grades of sheet metals with high performances, such as combined ductility and strength, greatly improves the quality of the final products. Examples in the automotive industry include crash resistance and weight reduction to improve fuel efficiency. However, several undesirable phenomena are observed during the forming of such materials which compromise the formed part. Springback is one of the most significant drawbacks of these new sheet materials. This phenomenon has been investigated by many authors (e.g. Mattiasson et al., 1995; Pourboghrat and Chu, 1995; He and Wagoner, 1996; Carden et al., 2002; Li et al., 2002; Lee et al., 2005a, 2005b). In studying springback, much attention is given to its accurate evaluation whether by analytical computations or by finite element (FE) simulations. In this context, it is important to have a deep understanding of the influence of all factors (process, numerical, behavior). This understanding permits the identification of the predominant factors and how their interaction affects the final shape prediction.

Proper material behavior description has been identified in many works as one of the most important factors to consider for springback predictions via FE simulations. During the last decades, many models have been formulated at different scales (micro, meso and macroscopic) to describe the elastoplastic behavior of sheet metals. In sheet metal forming simulations, phenomenological (macroscopic scale) models are widely used, since they present a good compromise between the model accuracy and process simulation computation time. The mathematical theory of elastoplasticity is now well understood since the works of Hill (1950) and more recently those of Lemaitre and Chaboche (1990), Khan and Huang (1995) and others. These works give a general framework for the development of more advanced behavior models (initial and induced anisotropy).

The description of the hardening during complex loading paths has received considerable attention. For simple applications, the Swift's and Voce's laws are widely used to reproduce the isotropic hardening during monotonic loading paths. However, strain-path changes induce more complex phenomena which must be considered in the constitutive model. To reproduce the decrease in flow stress upon reverse loading (Bauschinger effect), Prager (1956) and Ziegler (1959) proposed linear evolution laws for back-stress - the variable describing the kinematic hardening. Armstrong and Frederick (1966) proposed a non linear evolution for this type of hardening, which has been successively improved to reproduce the Bauschinger effect, the ratcheting effect in fatigue etc. (Chaboche, 1986, 1989; Lemaitre and Chaboche, 1990; Ohno and Kachi, 1986; Ohno and Wang, 1993a, 1993b; Abdel-Karim and Ohno, 2000; Geng, 2000; Gau and Kinzel, 2001). Several back-stress variables with different evolution laws are often combined in these models. Chun et al. (2002a, 2002b) further improved the cyclic hardening model of Chaboche by considering different back-stress evolution laws for the monotonic loading and the reverse loading. In their approach, several back-stress laws are deactivated upon loading reversal in order to obtain a different flow stress saturation level. Chung et al. (2005), Geng et al. (2002), Geng and Wagoner (2002), Khan and Huang (1995), proposed modified versions of Chaboche models by considering some of the kinematic hardening parameters as functions of the effective 
plastic strain. Recently, Choi et al. (2006a, 2006b) added rotational hardening for the description of the multi-axial elastoplastic behavior.

Since hardening is essentially due to the dislocation microstructure and its evolution, attempts have been made to describe their effect on hardening at a macroscopic scale. Following this approach, Teodosiu and Hu (1995, 1998), Teodosiu (1997) proposed a microstructure-based model representing not only the monotonic or reverse loading, but also the whole range between the two, including the particular case of orthogonal strainpath. More precisely, the introduction of physically-motivated internal variables that describe the evolution of the persistent dislocation structures allowed new transient phenomena to be modeled. Stagnation, softening and rapid change in work hardening rate - as observed during abrupt, two-stage sequential rheological tests - for a wide range of sheet metals are well described with this model (Bouvier et al., 2003).

In the current paper, the plastic anisotropy induced by hardening has been modeled by the microstructural hardening model proposed by Teodosiu and $\mathrm{Hu}$ (1998). The model can be coupled with any yield potential to take into account initial anisotropy. The corresponding constitutive equations are implemented in the ABAQUS software using the return mapping algorithm. A combined Euler implicit and asymptotic integration scheme is used to evaluate internal state variable evolution. The classical cyclic hardening model of Chaboche, combining the Armstrong-Frederick's and Voce's laws, is deduced as a particular case by setting some material parameters of the Teodosiu-Hu model to zero.

Springback is considered in this work, as one of the most challenging simulation tasks, in order to investigate the impact of hardening models on the accuracy of sheet metal forming simulations. In parallel, the sensitivity of springback predictions to purely numerical aspects of the simulation is also addressed. The well-known stripdrawing test is used for this analysis. Two different die and strip geometries and two materials - each of them being described with the two hardening models - have been used to generate the results discussed in the paper.

The paper is structured in three parts. In the first one, a general framework of the constitutive equations is developed, based on the large deformation theory. Both hardening models considered in this paper are shown to fit this general framework. The second part deals with the numerical implementation of these models. The main aspects of the time integration algorithm are developed and the consistent tangent modulus is given in a compact form for each model. In the last part, the FE predictions of these models are analysed. Their respective potential to reproduce transient features of the hardening is investigated by means of rheological test simulations. Finally, a detailed springback analysis is performed using several geometries, holding forces, and materials.

\section{Constitutive equations}

The phenomenological elastoplastic modeling adopted here is rate independent (without viscous effects) and restricted to cold deformation. The material is initially stress-free (well annealed state) and homogeneous. As previously mentioned, the two hardening models employed fall under the category of classic elastoplastic 
phenomenological modeling. They utilize a hypo-elastic law defining the stress rate with respect to the elastic strain rate, a yield criterion delimiting the elastic zone, a plastic flow rule and a set of internal state variable evolution laws defining the work hardening during plastic deformation.

\subsection{Kinematics}

Since the sheet undergoes large deformations in metal forming, the elastoplastic behavior is described by rate constitutive equations. In order to achieve material objectivity, objective rates must be used. Consequently, the constitutive models are often written in a convenient frame in order to simplify their formulation and further, their FE implementation. A short description of the large deformation theory used in many FE codes is recalled here.

The kinematics of large elastoplastic deformation are based on the multiplicative decomposition of the deformation gradient $\mathbf{F}$ into plastic part $\mathbf{F}^{p}$ and elastic part $\mathbf{F}^{e}$, i.e.

$$
\mathbf{F}=\mathbf{F}^{e} \cdot \mathbf{F}^{p}, \quad \mathbf{F}^{e} \cong(\mathbf{1}+\mathbf{e}) \cdot \mathbf{R}
$$

In the last equation, the elastic strains are considered small with respect to unity, which is always true for sheet metals; nevertheless, large rotations are rigorously considered. Here, $\mathbf{1}$ is the second order identity tensor, $\mathbf{e}$ is the symmetric tensor of small elastic strain $(|\mathbf{e}|<<1)$, and $\mathbf{R}$ is the rotation tensor. The velocity gradient $\mathbf{L}$, the strain rate tensor $\mathbf{D}$ and the material spin $\mathbf{W}$ are given by:

$$
\begin{gathered}
\mathbf{L}=\dot{\mathbf{F}} \cdot \mathbf{F}^{-1}=\dot{\mathbf{R}} \cdot \mathbf{R}^{T}+\stackrel{\circ}{\mathbf{e}}+\mathbf{R} \cdot \dot{\mathbf{F}}^{p} \cdot\left(\mathbf{F}^{p}\right)^{-1} \cdot \mathbf{R}^{T} \\
\mathbf{D}=\frac{1}{2}\left(\mathbf{L}+\mathbf{L}^{T}\right)=\stackrel{\circ}{\mathbf{e}}+\mathbf{D}^{p}, \quad \mathbf{W}=\frac{1}{2}\left(\mathbf{L}-\mathbf{L}^{T}\right)
\end{gathered}
$$

where $\mathbf{e}$ and $\mathbf{D}^{p}$ are the objective time derivative of the elastic strain tensor and the plastic strain rate, respectively, given by the following expressions:

$$
\stackrel{\mathbf{e}}{=} \dot{\mathbf{e}}+\mathbf{e} \cdot \dot{\mathbf{R}} \cdot \mathbf{R}^{T}-\dot{\mathbf{R}} \cdot \mathbf{R}^{T} \cdot \mathbf{e}, \quad \mathbf{D}^{p}=\mathbf{R} \cdot\left[\dot{\mathbf{F}}^{p} \cdot\left(\mathbf{F}^{p}\right)^{-1}\right]^{s y m} \cdot \mathbf{R}^{T}
$$

where []$^{s y m}$ designates the symmetric part.

The elastoplastic constitutive equations require the use of such objective rates. A very convenient approach consists of reformulating these equations in terms of rotationcompensated variables. More precisely, if $\mathbf{A}$ and $\mathbf{S}$ designate second and fourth order tensors, respectively, the corresponding rotation-compensated tensors are defined as follows:

$$
\hat{A}_{i j}=\Re_{k i} \Re_{l j} A_{k l}, \quad \hat{S}_{i j k l}=\Re_{p i} \Re_{q j} \Re_{r k} \Re_{s l} S_{p q r s}
$$


where $\Re$ is an orthogonal rotation matrix, generated by a skew-symmetric spin tensor $\mathbf{\Omega}$ using $\dot{\mathfrak{R}} \cdot \mathfrak{R}^{T}=\mathbf{\Omega}$.

The main interest of this approach is that objective derivatives defined like in Eq. (3) by $\dot{\mathbf{A}}=\dot{\mathbf{A}}+\mathbf{A} \cdot \boldsymbol{\Omega}-\boldsymbol{\Omega} \cdot \mathbf{A}$, are simply related to the material time derivatives of their rotation-compensated counterparts via equations similar to Eq. (4.a), i.e.

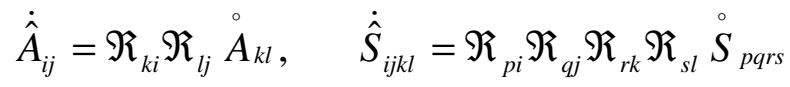

For example, the Jaumann derivative is obtained by setting $\boldsymbol{\Omega}=\mathbf{W}$, while using $\Re=\mathbf{R}$ leads to the Green-Naghdi derivative. Jaumann rates are considered throughout the present work.

Note that this transformation preserves the norms, defined by $|\mathbf{A}|=\sqrt{A_{i j} A_{i j}}$ and $|\mathbf{S}|=\sqrt{S_{i j k l} S_{i j k l}}$. In the following sections, all variables are written in the co-rotational frame, that is to say, with the rotation-compensated variables. Consequently, simple time derivatives are involved in the constitutive equations, making them identical in form to a small-strain formulation. For simplicity the superposed hat $(\wedge)$ is omitted thereafter.

\subsection{Constitutive model framework}

The Cauchy stress rate $\dot{\boldsymbol{\sigma}}$ is given by the hypo-elastic law

$$
\dot{\boldsymbol{\sigma}}=\mathbf{C}: \mathbf{D}^{e}=\mathbf{C}:\left(\mathbf{D}-\mathbf{D}^{p}\right)
$$

where $\mathbf{C}$ is the fourth order tensor of the elastic constants, while $\mathbf{D}$ and $\mathbf{D}^{e}$ are the strain rate and elastic strain rate tensors, respectively. The plastic strain rate tensor $\mathbf{D}^{p}$ is given by the associated flow rule:

$$
\mathbf{D}^{p}=\dot{\lambda} \frac{\partial F}{\partial \boldsymbol{\sigma}}=\dot{\lambda} \mathbf{V}
$$

where $\mathbf{V}$ is the flow direction normal to the yield surface defined by the potential $F$, and $\dot{\lambda}$ is the plastic multiplier to be determined from the loading-unloading criterion. This can be expressed in Kuhn-Tucker form as

$$
\begin{gathered}
F=\bar{\sigma}\left(\boldsymbol{\sigma}^{\prime}-\mathbf{X}\right)-Y \leq 0 \\
\dot{\lambda} \geq 0 \\
F \dot{\lambda}=0
\end{gathered}
$$

where $\bar{\sigma}$ is the equivalent stress, a function of $\boldsymbol{\sigma}^{\prime}$ (the deviatoric part of the Cauchy stress) and the back-stress $\mathbf{X}$, whereas $Y$ is the size of the yield surface. 
If the hardening is governed by the rate equations of the form

$$
\dot{Y}=H_{Y} \dot{\lambda}, \quad \dot{\mathbf{X}}=\mathbf{H}_{\mathbf{x}} \dot{\lambda}
$$

the consistency condition $\dot{F}=0$ leads to

$$
\dot{\lambda}=\frac{\mathbf{V}: \mathbf{C}: \mathbf{D}}{\mathbf{V}: \mathbf{C}: \mathbf{V}+\mathbf{V}: \mathbf{H}_{\mathbf{x}}+H_{Y}}
$$

This expression can be used for any particular yield surface and hardening laws in the form of Eq. (8). The analytical elastoplastic tangent modulus can then be derived as

$$
\mathbf{C}^{e p}=\mathbf{C}-\alpha \frac{(\mathbf{C}: \mathbf{V}) \otimes(\mathbf{V}: \mathbf{C})}{\mathbf{V}: \mathbf{C}: \mathbf{V}+\mathbf{V}: \mathbf{H}_{\mathbf{x}}+H_{Y}}
$$

where $\alpha=1$ for plastic loading and 0 otherwise. If the tensor $\mathbf{C}$ is isotropic, these expressions are further simplified giving

$$
\dot{\lambda}=\frac{2 G \mathbf{V}: \mathbf{D}}{2 G|\mathbf{V}|^{2}+\mathbf{V}: \mathbf{H}_{\mathbf{x}}+H_{Y}}
$$

and

$$
\mathbf{C}^{e p}=\mathbf{C}-\alpha \frac{4 G^{2} \mathbf{V} \otimes \mathbf{V}}{2 G|\mathbf{V}|^{2}+\mathbf{V}: \mathbf{H}_{\mathbf{X}}+H_{Y}}
$$

where $G$ is the elastic shear modulus.

So far, the material model has been kept in a general analytical form. The yield function is defined by the equivalent stress $\bar{\sigma}$ and its gradient $\mathbf{V}$, while the hardening is defined by $H_{Y}$ and $\mathbf{H}_{\mathbf{x}}$. It is important to note that all internal state variables (denoted by the vector $\mathbf{y}$ ) are governed by rate equations of the type

$$
\dot{\mathbf{y}}=\mathbf{H}_{\mathbf{y}} \dot{\lambda}
$$

Thus, a general time integration scheme can be built for any hardening model or yield function (see section 3.1). Several yield functions can be introduced in order to model the initial anisotropy; however, since the paper focuses on the hardening models, the quadratic Hill' 48 yield surface (Hill, 1950) is adopted here. This model is defined by

$$
\bar{\sigma}=\sqrt{\mathbf{T}: \mathbf{M}: \mathbf{T}}
$$


where $\mathbf{T}=\boldsymbol{\sigma}^{\prime}-\mathbf{X}$ is the effective stress and $\mathbf{M}$ is a fourth order tensor containing the six anisotropy coefficients of Hill.

\subsection{Hardening models}

As for the initial plastic anisotropy, several hardening models can be introduced in the framework of phenomenological elastoplasticity. The classical cyclic hardening model of Chaboche and the dislocation-based microstructural model of Teodosiu and $\mathrm{Hu}(1998)$ are considered in this work.

\subsubsection{Classical cyclic hardening model}

This hardening model (see e.g. Chaboche, 1991) describes isotropic and kinematic hardening with two internal state variables. These variables are a scalar variable $R$, describing the isotropic hardening, and a second order tensorial variable $\mathbf{X}$, describing the kinematic hardening. The variable $\mathbf{X}$ allows reproduction of the Bauschinger effect.

The isotropic hardening evolution gives the variation of the yield surface size $Y$ by

$$
Y=Y_{0}+R
$$

where $Y_{0}$ is the initial value of the yield stress. The evolution of $R$ is given by the Voce's law:

$$
\dot{R}=C_{R}\left(R_{\text {sat }}-R\right) \dot{\lambda}=H_{R} \dot{\lambda}
$$

where $C_{R}$ characterizes the saturation rate of $R$ and $R_{\text {sat }}$ is its saturation value.

The kinematic hardening gives the translation of the yield surface in the stress space. Its evolution law is given by an Armstrong-Frederick-type law described by:

$$
\dot{\mathbf{X}}=C_{X}\left(X_{s a t} \mathbf{n}-\mathbf{X}\right) \dot{\lambda}=\mathbf{H}_{\mathbf{x}} \dot{\lambda}
$$

where $C_{X}$ and $X_{\text {sat }}$ are material parameters characterizing the saturation rate and saturation value of $\mathbf{X}$, respectively, while $\mathbf{n}=\mathbf{T} / \bar{\sigma}$ is the saturation direction. Note that $\mathbf{n}$ is parallel to the plastic strain rate tensor for the von Mises yield surface, and that the scalar function $H_{Y}$ in Eqs. (8), (9) and (10) is found from

$$
H_{Y}=H_{R}=C_{R}\left(R_{s a t}-R\right)
$$

This model is considered as a reference in the current work, since it is widely used in literature to take into account the Bauschinger effect in metal forming simulations. Moreover, this model is available in many commercial FE codes, including Abaqus. 


\subsubsection{Dislocation-based microstructural model}

This advanced hardening model is able to reproduce not only the Bauschinger effect but also other transient hardening phenomena observed during two-stage sequential rheological tests. It is based on physical considerations, mainly the description of the evolution of the so-called planar persistent dislocation structures (PPDS) and their contribution to the hardening of the material. Proper description of PPDS evolution mechanisms will reproduce the elementary transient phenomena observed on stressstrain curves.

This model involves four internal state variables: $R, \mathbf{X}, \mathbf{S}$ and $\mathbf{P}$. The variable $R$ is a scalar, while $\mathbf{X}$ and $\mathbf{P}$ are second order tensors and $\mathbf{S}$ is a fourth order tensor. Note that $R, \mathbf{X}$ and $\mathbf{S}$ have units of stress and $\mathbf{P}$ is unitless. The presentation of the model follows the original paper of Teodosiu and $\mathrm{Hu}$ (1998).

The yield surface size is given as function of $R$ and $\mathbf{S}$ by

$$
Y=Y_{0}+R+f|\mathbf{S}|
$$

where $R$ describes the contribution of the randomly distributed dislocations to the isotropic hardening. Its evolution law is given by the Voce's law, Eq. (16). The term $f|\mathbf{S}|$ represents the affect of PPDS on isotropic hardening, where $\mathbf{S}$ describes the directional strength of the PPDS and $f$ is a material parameter.

The kinematic hardening evolution law, described by the back-stress variable $\mathbf{X}$, is given by Eq. (17). Nevertheless, the saturation value $X_{\text {sat }}$ is no longer a material parameter in this model, but a function of the internal state variable $\mathbf{S}$. This dependency of $X_{\text {sat }}$ on the $\mathbf{S}$ variable is assumed of the form

$$
X_{\text {sat }}=X_{0}+(1-f)|\mathbf{S}| \sqrt{r+(1-r) \beta_{S}^{2}}
$$

where $X_{0}$ is the initial value of $X_{\text {sat }}$ and $r$ is a material parameter. The ratio $\beta_{S}=S_{D} / \mathbf{S}$ is a measure of the change in orientation of the current strain rate tensor with respect to the PPDS (Teodosiu and $\mathrm{Hu}, 1995$ ). This parameter is therefore considered to be an indicator of strain-path change. It evolves between 0 (orthogonal loading) and 1 (monotonic or reverse loading).

Experimental observations indicate that the PPDS associated with the current direction of the strain rate evolve quite differently from the rest of the PPDS during strain-path change. The variable $\mathbf{S}$ is therefore decomposed into two parts: $S_{D}$ (scalar) and $\mathbf{S}_{L}$ (fourth order tensor), where $S_{D}$ represents the strength associated with the currently active slip systems, whereas $\mathbf{S}_{L}$ is associated with the latent part of the PPDS. The decomposition of $\mathbf{S}$ takes the following form

$$
S_{D}=\mathbf{N}: \mathbf{S}: \mathbf{N}, \quad \mathbf{S}_{L}=\mathbf{S}-S_{D} \mathbf{N} \otimes \mathbf{N}
$$


where $\mathbf{N}=\mathbf{D}^{p} /\left|\mathbf{D}^{P}\right|$ and represents the plastic strain rate direction. The evolution laws of $S_{D}$ and $\mathbf{S}_{L}$ are given by

$$
\begin{gathered}
\dot{S}_{D}=C_{S D}\left[g\left(S_{s a t}-S_{D}\right)-h S_{D}\right] \dot{\lambda}=H_{S_{D}} \dot{\lambda} \\
\dot{\mathbf{S}}_{L}=-C_{S L}\left(\frac{\left|\mathbf{S}_{L}\right|}{S_{\text {sat }}}\right)^{n_{L}} \mathbf{S}_{L} \dot{\lambda}=\mathbf{H}_{\mathbf{S}_{L}} \dot{\lambda}
\end{gathered}
$$

where $S_{s a t}$ and $C_{S D}$ are the saturation value and rate of $S_{D}$, respectively, while $C_{S L}$ and $n_{L}$ characterize the saturation rate of $\mathbf{S}_{L}$. The functions $g$ and $h$ have been introduced in order to capture transient hardening after a change in strain-path. Their assumed mathematical forms are given below:

$$
g=\left\{\begin{array}{cc}
1-\frac{C_{P}}{C_{S D}+C_{P}}\left|\frac{S_{D}}{S_{s a t}}-\mathbf{P}: \mathbf{N}\right| & \text { if } \mathbf{P}: \mathbf{N} \geq 0 \\
(1+\mathbf{P}: \mathbf{N})^{n_{p}}\left(1-\frac{C_{P}}{C_{S D}+C_{P}} \frac{S_{D}}{S_{s a t}}\right) & \text { otherwise } \\
h=\frac{1}{2}\left(1-\frac{\mathbf{X}: \mathbf{N}}{X_{s a t} \mathbf{n}: \mathbf{N}}\right) &
\end{array}\right.
$$

In these expressions $n_{p}$ is a material parameter and $\mathbf{P}$ is the internal state variable describing the polarity of the PPDS. Its evolution law is given by

$$
\dot{\mathbf{P}}=C_{p}(\mathbf{N}-\mathbf{P}) \dot{\lambda}
$$

where $C_{P}$ characterizes the polarization rate of the PPDS.

From Eqs. (21) and (22), one can obtain the time derivative of the norm, $|\mathbf{S}|=\sqrt{\left|\mathbf{S}_{L}\right|^{2}+S_{D}^{2}}$, of the $\mathbf{S}$ tensor as

$$
|\mathbf{S}|=\frac{1}{|\mathbf{S}|}\left[H_{S_{D}} S_{D}-C_{S L}\left(\frac{\left|\mathbf{S}_{L}\right|}{S_{\text {sat }}}\right)^{n_{L}}\left|\mathbf{S}_{L}\right|^{2}\right] \dot{\lambda}=H_{|\mathbf{S}|} \dot{\lambda}
$$

Thus the scalar function $H_{Y}$ in Eqs. (8), (9) and (10) is deduced for this model as

$$
H_{Y}=H_{R}+f H_{|\mathbf{S}|}
$$




\section{Numerical implementation}

The elastoplastic models introduced above have been implemented in the static implicit code ABAQUS/Standard. At each equilibrium iteration, a displacement increment is predicted in each node of the mesh. From this, the kinematics equations are employed to calculate the strain increment at each integration point of the finite elements. These steps are carried out by the FE code, so only the resolution of the constitutive equations (state update) needs to be performed to verify the equilibrium state at the end of the loading increment. In the following, we develop the state update methodology as well as the consistent tangent modulus, necessary to achieve the equilibrium state at the end of each loading increment.

\subsection{Discrete form of the constitutive equations}

In the previous section, Eqs. (5) to (7) and (13) were shown to completely define the constitutive model. The FE implementation of such a model requires the numerical integration of these equations over a time increment, from a known state at time $t_{n}$ to the unknown state at $t_{n+1}-$ given the total strain increment $\Delta \boldsymbol{\varepsilon}$. The most widely used method is the fully implicit, backward Euler integration scheme (e.g. Hughes, 1984; Simo and Taylor, 1985; Ortiz and Popov, 1985). This method leads to the following discrete form of the constitutive equations

$$
\begin{gathered}
\Delta \boldsymbol{\sigma}=\mathbf{C}:\left(\Delta \boldsymbol{\varepsilon}-\Delta \boldsymbol{\varepsilon}^{p}\right) \\
\Delta \boldsymbol{\varepsilon}^{p}=\Delta \lambda \mathbf{V}_{n+1} \\
F_{n+1}=\bar{\sigma}\left(\mathbf{T}_{n+1}\right)-Y\left(\mathbf{y}_{n+1}\right)=0 \\
\Delta \mathbf{y}=\mathbf{h}_{1}\left(\mathbf{y}_{n+1}, \boldsymbol{\sigma}_{n+1}, \Delta \lambda\right)
\end{gathered}
$$

where $\Delta(\cdot)=(\cdot)_{n+1}-(\cdot)_{n}$, while $\mathbf{h}_{1}$ is a function that will be defined in section 3.2. This is a system of four equations (tensorial and scalar) with the four unknowns $\boldsymbol{\sigma}_{n+1}, \Delta \boldsymbol{\varepsilon}^{p}$, $\Delta \lambda$ and $\mathbf{y}_{n+1}$. A general method for the direct resolution of this kind of system has been proposed by Keavey (2001). Nevertheless, no applications of this approach have been made where the number of internal variables becomes large (e.g. more than fifty scalar equations for the Teodosiu-Hu model). In practice, this would imply a significant increase in computation time as well as possible convergence difficulties due to the nonlinearity of the constitutive equations. Consequently, most existing implementations (Hartmann and Haupt, 1993; Alves, 2003; De Montleau et al., 2004) attempt to reduce the size of the nonlinear system to be solved. The nonlinearity of advanced elastoplastic constitutive models often requires the development of specific time-integration algorithms, including e.g. sub-stepping (Yoon et al., 1999; Abdel-Karim, 2005; Khoei and Jamali, 2005). Generally convergent algorithms for elastoplasticity have been recently proposed (Armero and Pérez-Foguet, 2002; Pérez-Foguet and Armero, 2002), which avoid the need for specific algorithms in most cases. 
Several methods can be adopted for the time integration of the internal state variables evolution laws such as Eq. (13). Let us restrict our development to a particular case where the discrete form $\mathbf{y}_{n+1}$, given in Eq. (27.d), can be written under the explicit form

$$
\mathbf{y}_{n+1}=\mathbf{h}_{2}\left(\mathbf{T}_{n+1}, \Delta \lambda\right)
$$

More details will be given in the next section.

The remaining equations - i.e. Eqs. (27.a) to (27.c) can now be rearranged and further simplified without any assumption, by considering the following steps:

- $\quad$ Take the deviatoric part of (27.a);

- Substitute $\Delta \boldsymbol{\varepsilon}^{p}$ from (27.b) to (27.a);

- Substitute $\mathbf{y}_{n+1}$ from (28) to (27.c);

- Explicitly write $\mathbf{C}$ for the case of linear, isotropic elasticity (although this is not required for the current development, this is almost always the case and it simplifies the final equations).

Moreover, a careful analysis of the equations in section 2 suggests $\mathbf{T}$ as unknown in the final system, instead of $\boldsymbol{\sigma}$. Indeed, $\boldsymbol{\sigma}$ enters Eqs. (27.b) to (27.d) exclusively as part of $\mathbf{T}$; equation (27.a) can be easily adapted to this change of variable.

With these transformations, Eq. (27) becomes

$$
\begin{gathered}
\mathbf{T}_{n+1}-\boldsymbol{\sigma}_{n}^{\prime}-2 G \Delta \boldsymbol{\varepsilon}^{\prime}+2 G \Delta \lambda \mathbf{V}_{n+1}\left(\mathbf{T}_{n+1}\right)+\mathbf{X}_{n+1}\left(\mathbf{T}_{n+1}, \Delta \lambda\right)=\mathbf{0} \\
F_{n+1}=\bar{\sigma}\left(\mathbf{T}_{n+1}\right)-Y\left(\mathbf{y}_{n+1}\left(\mathbf{T}_{n+1}, \Delta \lambda\right)\right)=0
\end{gathered}
$$

Since the expressions of the internal state variables can all be substituted here from Eq. (28), it follows that Eq. (29) provides the final, reduced form of the constitutive model in its discrete form. The system size is reduced to six (five components of the symmetric, deviatoric tensor $\mathbf{T}$, plus $\Delta \lambda$ ) in a general 3-D analysis. After solving this system by the Newton-Raphson method and then updating the internal state variables using Eq. (28), the Cauchy stress is updated using the relation:

$$
\boldsymbol{\sigma}_{n+1}=\boldsymbol{\sigma}_{n+1}^{\prime}+\sigma_{n+1}^{s} \mathbf{1}
$$

with $\sigma_{n+1}^{s}$ (spherical part of the Cauchy stress tensor) and $\boldsymbol{\sigma}_{n+1}^{\prime}$ is deduced from the relations

$$
\begin{gathered}
\sigma_{n+1}^{s}=\frac{1}{3} \operatorname{tr}\left(\boldsymbol{\sigma}_{n+1}\right)=\frac{1}{3} \operatorname{tr}\left(\boldsymbol{\sigma}_{n}\right)+\operatorname{Ktr}(\Delta \boldsymbol{\varepsilon}) \\
\boldsymbol{\sigma}_{n+1}^{\prime}=\mathbf{T}_{n+1}+\mathbf{X}_{n+1}
\end{gathered}
$$

where $K$ is the elastic bulk modulus. 


\subsection{Update of the internal variables}

In the framework of the implicit integration, several choices are available to integrate the evolution equations of the internal state variables, $\dot{\mathbf{y}}=\mathbf{h}(\boldsymbol{\sigma}, \mathbf{y}) \dot{\boldsymbol{\lambda}}$. The Backward Euler scheme leads to an update equation of the form $\Delta \mathbf{y}=\mathbf{h}\left(\boldsymbol{\sigma}_{n+1}, \mathbf{y}_{n+1}\right) \Delta \lambda$. Applying this scheme to Eq. (17), for example, generates the following update equation for the back-stress $\mathbf{X}$ :

$$
\mathbf{X}_{n+1}=\frac{1}{1+C_{X} \Delta \lambda}\left(\mathbf{X}_{n}+C_{X} X_{n+1}^{s a t} \Delta \lambda \mathbf{n}_{n+1}\right)
$$

Nevertheless, for some evolution equations, an analytical integration can be performed. This is the case for a particular form of saturation-type equations, where an accurate asymptotic integration has been proposed (Freed and Walker, 1992) and has been adopted in many FE implementations (e.g. Chaboche and Cailletaud, 1996; Abaqus, 2003). For many other cases, a similar semi-analytical integration can be performed under some hypotheses and the result still can be more accurate than the Backward Euler formula (Hoferlin et al., 2001; Balan and Teodosiu, 1999). Consider Eq. (17) again as an example. It is possible to separate variables $\mathbf{X}$ and $\lambda$ and analytically integrate this equation, provided that $\mathbf{n}$ is considered constant over the increment (and equal to its value at the end of the increment). This hypothesis is consistent with Eq. (27.b), where $\mathbf{V}$ is also considered constant and equal to its value at the end of the increment. By applying this approach, explicit and independent update equations can be obtained for the Teodosiu-Hu model, under the following form:

$$
\begin{gathered}
\mathbf{X}_{n+1}=\mathbf{X}_{n} e^{-C_{X} \Delta \lambda}+X_{n+1}^{s a t} \mathbf{n}_{n+1}\left(1-e^{-C_{X} \Delta \lambda}\right) \\
R_{n+1}=R_{n}+\left(R_{s a t}-R_{n}\right)\left(1-e^{-C_{R} \Delta \lambda}\right) \\
\mathbf{P}_{n+1}=\mathbf{P}_{n}+\left(\mathbf{N}_{n+1}-\mathbf{P}_{n}\right)\left(1-e^{-C_{P} \Delta \lambda}\right) \\
S_{n+1}^{D}=S_{n}^{D} e^{-C_{S D}(g+h) \Delta \lambda}+S_{s a t} \frac{g}{(g+h)}\left(1-e^{-C_{S D}(g+h) \Delta \lambda}\right) \\
\mathbf{S}_{n+1}^{L}=\left|\mathbf{S}_{n+1}^{L}\right| \frac{\mathbf{S}_{n}^{L}}{\left|\mathbf{S}_{n}^{L}\right|}=\left(\left|\mathbf{S}_{n}^{L}\right|^{-n_{L}}+n_{L} \frac{C_{S L}}{S_{s a t}^{n_{L}}} \Delta \lambda\right)^{-\frac{1}{n_{L}}} \frac{\mathbf{S}_{n}^{L}}{\left|\mathbf{S}_{n}^{L}\right|} \\
\mathbf{S}_{n+1}=\mathbf{S}_{n+1}^{L}+S_{n+1}^{D} \mathbf{N}_{n+1} \otimes \mathbf{N}_{n+1} \\
|\mathbf{S}|_{n+1}=\sqrt{\left|\mathbf{S}_{n+1}^{L}\right|^{2}+S_{n+1}^{D}{ }^{2}}
\end{gathered}
$$

Note that the functions $g$ and $h$ in the expression for $S_{D}$ as well as $X_{\text {sat }}$ are assumed equal to their values of the beginning of the increment. These approximations are suggested by the structure of the model and the phenomena it describes. Indeed, from physical and experimental observations (Teodosiu and Hu, 1998; Teodosiu, 2003), the evolutions of $S_{D}$ and $X_{\text {sat }}$ are known to be much slower than those of $\mathbf{D}^{p}, \boldsymbol{\sigma}$ or $\mathbf{X}$ which are evaluated implicitly, along with all other internal variables. From a numerical perspective, these approximations allow the Teodosiu-Hu model to fit the requirements 
of Eq. (28), and reduce the size of the nonlinear system to be solved by an order of magnitude.

\subsection{Consistent tangent modulus}

The convergence rate of the Newton-Raphson resolution of the equilibrium equations depends on the form of the tangent modulus to be introduced as stated in several works, e.g. (Hughes, 1984) and (Simo and Taylor, 1985). This consistent modulus should be derived from the constitutive equations used to update the state variables. It relates linearly the variation of the Cauchy stress increment to the infinitesimal variation of the strain increment that produced it, i.e.

$$
D(\Delta \boldsymbol{\sigma})=\mathbf{C}^{\text {cons }}: D(\Delta \boldsymbol{\varepsilon})
$$

To compute this modulus, the incremental forms of the hypoelastic law and the plastic flow rule, i.e. Eqs. (27.a) and (27.b) are differentiated. This gives the following equations:

$$
\begin{gathered}
D(\Delta \boldsymbol{\sigma})=\mathbf{C}:\left[D(\Delta \boldsymbol{\varepsilon})-D\left(\Delta \boldsymbol{\varepsilon}^{\mathbf{p}}\right)\right] \\
D\left(\Delta \boldsymbol{\varepsilon}^{p}\right)=D(\Delta \lambda \mathbf{V})=D(\Delta \lambda) \mathbf{V}+\Delta \lambda D(\mathbf{V})
\end{gathered}
$$

where

$$
D(\mathbf{V})=\frac{\partial \mathbf{V}}{\partial \mathbf{T}}: D(\mathbf{T})=\mathbf{Q}: D(\mathbf{T})
$$

and, from the differentiation of Eq. (29.b):

$$
D(\Delta \lambda)=\frac{1}{H}\left(\mathbf{V}-\frac{\partial Y}{\partial \mathbf{T}}\right): D(\mathbf{T})
$$

with

$$
H=\frac{\partial Y}{\partial \Delta \lambda}=\frac{\partial R}{\partial \Delta \lambda}+f \frac{\partial|\mathbf{S}|}{\partial \Delta \lambda}
$$

Replacing $D(\Delta \lambda)$ and $D(\mathbf{V})$ by their corresponding expressions in Eq. (35.b) yields a linear relationship between $D\left(\Delta \boldsymbol{\varepsilon}^{p}\right)$ and $D(\mathbf{T})$, i.e.

$$
D\left(\Delta \boldsymbol{\varepsilon}^{p}\right)=\left[\frac{1}{H} \mathbf{V} \otimes\left(\mathbf{V}-\frac{\partial Y}{\partial \mathbf{T}}\right)+\Delta \lambda \mathbf{Q}\right]: D(\mathbf{T})
$$

With the differentiation of Eq. (29.a) and after some rearranging, a linear relation is obtained between $D(\mathbf{T})$ and $D(\Delta \boldsymbol{\varepsilon})$, i.e. 


$$
D(\mathbf{T})=2 G \boldsymbol{\Lambda}^{-1}: D(\Delta \boldsymbol{\varepsilon})
$$

with

$$
\boldsymbol{\Lambda}=\mathbf{I}_{4}^{\prime}+2 G\left[\frac{1}{H} \mathbf{V} \otimes\left(\mathbf{V}-\frac{\partial Y}{\partial \mathbf{T}}\right)+\Delta \lambda \mathbf{Q}\right]+\frac{\partial \mathbf{X}}{\partial \mathbf{T}}+\frac{1}{H} \frac{\partial \mathbf{X}}{\partial \Delta \lambda} \otimes\left(\mathbf{V}-\frac{\partial Y}{\partial \mathbf{T}}\right)
$$

where $\mathbf{I}_{4}^{\prime}$ is the fourth order symmetric and deviatoric identity tensor.

By replacing $D(\mathbf{T})$ by its expression in Eq. (39) and then $D\left(\Delta \boldsymbol{\varepsilon}^{p}\right)$ in Eq. (35.a), a linear relation is finally obtained between $D(\Delta \boldsymbol{\sigma})$ and $D(\Delta \boldsymbol{\varepsilon})$, thus defining thus the consistent tangent modulus, i.e.

$$
\mathbf{C}^{\text {cons }}=\frac{D(\Delta \boldsymbol{\sigma})}{D(\Delta \boldsymbol{\varepsilon})}=K(\mathbf{1} \otimes \mathbf{1})+2 G \mathbf{I}_{4}^{\prime}-\alpha 4 G^{2}\left[\frac{1}{H} \mathbf{V} \otimes\left(\mathbf{V}-\frac{\partial Y}{\partial \mathbf{T}}\right)+\Delta \lambda \mathbf{Q}\right]: \mathbf{\Lambda}^{-1}
$$

where $\alpha=1$ for elastoplastic loading and $\alpha=0$ otherwise.

Note that this consistent tangent modulus is evaluated with the updated variables.

\subsection{Time integration algorithm}

The return mapping algorithm, used by many authors and adopted here, assumes an elastic prediction and a plastic correction (when required). This allows for a straightforward implementation of the Backward Euler scheme and also provides an effective numerical counterpart of the loading-unloading conditions (Eq. (7)), as stated e.g. by Hughes (1984). The resulting constitutive algorithm is outlined in Table 1. This algorithm has been implemented in the FE code Abaqus, in the form of a UMAT routine. The cyclic hardening model is available in the commercial version of Abaqus and has been used here for validation, by means of one-element simulation of the tensile test. The corresponding stress-strain curves are plotted in Fig. 1. The present implementation not only accurately fits the reference Abaqus simulation, but this accuracy is achieved even for very large strain increments - up to $30 \%$ of strain in one single increment. Since the regular strain increments in a sheet forming simulation never exceed $1 \%$ strain, the accuracy of the presented time integration scheme is thus largely satisfactory.

\section{Applications}

In this section, the role played by the selected hardening models in the springback prediction is investigated. The Teodosiu-Hu model was originally developed for mild steels, which exhibit very typical transition zones after strain-path change: early yielding and large hardening rates, followed by distinct plateaux (or even softening after orthogonal strain-path change) - and finally resumption of hardening at larger strains. It is for these reasons that a mild steel sheet has been considered in this study. 
Nevertheless, the currently growing interest for the accurate prediction of springback mainly concerns sheet metals with increased light-weighting potential for the automotive industry, e.g. high strength steels. Thus a dual phase (DP) steel is also considered, since the Teodosiu-Hu model has been shown to successfully predict the dual phase steels hardening behavior (Haddadi et al., 2006). Dual phase steels exhibit both a considerable Bauschinger effect and an increased amount of springback as compared to mild steels. Lee et al. (2005b) have shown that the springback of dual phase steels is comparable to the one of aluminum alloys. This is due to their similar maximum yield stress vs. elastic modulus ratio. Although the accurate description of the hardening of steels requires combined isotropic-kinematic hardening models, their yield surface can be successfully described by the classical Hill' 48 yield function. It is important to note that the choice of the yield function (and its parameters) is a key factor for an accurate springback prediction when anisotropic, non-quadratic yield surfaces are required (Yoon et al., 1999, Geng and Wagoner 2002). Thus the choice of two steels is consistent with the focus on the influence of hardening.

Once a constitutive model has been chosen to describe a material behavior, the corresponding material parameters must be identified. Parameter identification for combined isotropic-kinematic hardening models is a challenging task since the required reverse straining of sheet materials leads rapidly to wrinkling when compressive stresses are developed. Numerous experimental procedures have been proposed for the parameter identification of isotropic-kinematic hardening models. Original tensioncompression tests for sheet materials have been proposed by Kuwabara et al. (1995) using fork-shaped dies and by Boger et al. (2005) and Lee et al. (2005a) using flat dies for lateral compression to prevent buckling. The shear test has been successfully used (Miyauchi, 1984a,b; Genevois, 1992; Rauch, 1998; Barlat et al., 2003) for reverse loading at large strains. An identification procedure for advanced hardening models has been proposed by Teodosiu and $\mathrm{Hu}$ (1998), Haddadi et al. (2001; 2006), Bouvier et al. (2006), based on shear and tensile tests. Complete sets of parameters obtained by this identification procedure have been published, for several steels and aluminum alloys. Parameters for advanced hardening models including those of Chaboche and Teodosiu are listed in several references.

The main interest of this identification approach is that large amounts of pre-strain (up to $30 \%$ in shear) are used in both uniaxial tension and simple shear, followed by large amounts of subsequent strain (typically 50\%) in simple shear. As we shall see later, these values are typical for the strip drawing simulations performed in the current work. Consequently, when the accurate description of the experimental tests is enforced during the parameter identification phase, almost the whole range of pre-strains and subsequent strains induced by our applications is considered.

For the purpose of the current work, the material parameters of a mild steel and a DP steel are selected from (Haddadi et al., 2003), which correspond to the two material models investigated. Table 2 shows the Hill' 48 initial anisotropy parameters of the two materials and Table 3 contains the corresponding hardening parameters - for both models.

In what follows, the respective ability of the two models to predict rheological strainpath changes is investigated. Whereas the impact of the yield surface on the simulations 
of sheet forming and particularly on springback is well recognized, different hardening models are often shown in literature to predict similar springback, when the compared models include both isotropic and kinematic hardening (3DS Benchmarks, 2002; Alves, 2003; Lee et al., 2005b). Two geometries of the strip-drawing test are used in order to address the usefulness of the Teodosiu-Hu model for springback simulations. The numerical efficiency and robustness of the numerical implementation is also addressed in the context of the draw-bend test simulations.

\subsection{Rheological tests simulation}

A set of typical rheological tests was simulated to illustrate the behavior reproduced by the two hardening models. These are monotonic tests (tensile or shear), reverse (Bauschinger) shear tests with several amounts of pre-stain and an orthogonal test (tension followed by shear). These tests have been used by Teodosiu and $\mathrm{Hu}$ (1998) to emphasize the main strain-path-change effects on hardening. All tests are considered along the rolling direction. Fig. 2 and 3 overview the capability of the two models to predict the transient behavior after abrupt strain-path change, represented in terms of true stress versus true strain for the uniaxial tensile tests and shear stress versus amount of shear for the shear tests. As underlined by Bouvier et al. (2003), the predictions of the two models differ especially in the transition zone after abrupt strain-path changes while their monotonic responses are almost identical. The Teodosiu-Hu model provides an improved capability to describe the real transient hardening behavior for a wide range of sheet metals (Teodosiu and $\mathrm{Hu}, 1995,1998$; Bouvier et al., 2003).

From Fig. 2 and 3, the comparison between the predictions of the microstructural and classical models shows several differences during strain-path change. For the mild steel (see Fig. 2a), the Teodosiu-Hu model exhibits rapid work hardening followed by stagnation and then resumption of work hardening after loading reversal. The plateau length increases with increasing pre-strain. We also note that the phenomenon of rapid hardening immediately followed by softening in the orthogonal test is well reproduced. As shown in Fig. 3a, these transient phenomena cannot be captured with the classical model. It is noteworthy that several evolutions of the classical model significantly improve its predictions. As far as reverse loading is concerned, most of the recent hardening models cited in section 1 (e.g. Abdel-Karim and Ohno, 2000; Geng, 2000; Chun et al., 2002a and 2002b, Chung et al., 2005, Geng et al., 2002, Geng and Wagoner, 2002) would provide a better fit of both the transient and the saturation zones. The models of Yoshida et al. (2002a, 2002b) also predict accurately the stagnation and resumption of strain hardening in reverse loading, while involving fewer parameters than the Teodosiu-Hu one. The later has nevertheless the ability to describe other strainpath changes - e.g. orthogonal - due to its more physical background.

The transient phenomena are less pronounced for the dual phase steel, as can be seen in Fig. 2b, since the work hardening at the beginning of the second path in the reverse test is less rapid and the plateau is shorter than for the mild steel. It is noteworthy that the length of this plateau also increases with the increase in pre-strain. In the orthogonal path, the shear flow stress does not exceed that of monotonic shear at the transient stage. Similar observations are found by Yoshida et al. (2002a and 2002b) on a cyclic loading by tension/compression. 
Clearly, the predictions of the two models differ under non-monotonic strain-paths, while they are similar under monotonic loading. Thus, their strain-path dependency will be the unique source of difference in sheet metal forming simulations, such as the strip drawing springback analysis hereafter.

\subsection{Springback simulations for the strip drawing test}

The strip drawing test (see Fig. 4) has been proposed as a springback benchmark test at the NUMISHEET'93 (1993) conference. Since then, this test has been recognized as a reference benchmark test both for the experimental investigation of metal sheets and also for the validation of numerical simulations (Mattiasson et al., 1995; He and Wagoner, 1996; Duffett et al., 2002; Sabourin et al., 2002; Lee et al., 2005b; Dongjuan et al., 2006; Firat, 2006). This test is used here to highlight the effect of the hardening models, combined with process and numerical parameters. Some of the most important geometrical characteristics of the test are the ratios $L / W, D / W$, and $T / R$, where $W$ is the punch width, $L$ is the initial strip length, $D$ is the drawing stroke, $T$ is the sheet thickness and $R$ is the punch/die radius. Springback is not only larger for larger values of these ratios, but the simulation is also more challenging and more sensitive to the modeling parameters. Two test geometries are investigated; their corresponding geometrical parameters are given in Table 4.

The numerical simulation of both the drawing and the springback steps is performed with the static implicit code Abaqus/Standard. Given the small radii/thickness ratios, the sheet is modeled with solid elements for a better accuracy. For computing time convenience, all the simulations are performed using plane strain solid elements. This choice is sufficient for comparison purposes. It is important to note, however, that for a more rigorous simulation, three-dimensional effects should also be taken into account. Also, the tools are simply modeled using rigid surfaces.

\subsection{1. "Smooth" test geometry}

Several physical and numerical factors have been identified in the literature to have a considerable influence on springback. A general sensitivity study has been realized in this work, using the so-called smooth geometry. Three of the most representative series of simulations are shown in Fig. 5. This figure illustrates the influence of the blank holding force (BHF), the hardening law and the finite element formulation.

The impact of the BHF as a process parameter is highlighted in Fig. 5a. The amount of springback is inversely proportional to the BHF. Indeed, the increase of the BHF increases the stretching force on the sheet which becomes the predominant loading as compared to bending. Consequently, the stress distribution is more uniform and the residual bending moment is smaller as the BHF increases. This means that upon unloading, springback should decrease with increased BHF. Experimental tests systematically confirm this tendency for different material grades (see e.g. Chu, 1991; NUMISHEET'93 Benchmark Problem, 1993; Kuwabara et al., 1996).

The effect of the hardening model is almost absent in this simulation. Even extreme cases of purely isotropic hardening law or purely kinematic have little impact. The parameters of these simplified models - described by Eqs. (16) and (17), respectively - 
have been identified using a single tensile stress-strain curve and the values are given in Table 5. Finally, the hardening curve has also been directly input in Abaqus as a table of stress-strain data - the simplest way to describe hardening in a FE code. As shown in Fig. 5b, and as pointed out by several authors (e.g. Bouvier et al., 2005; Lee et al., $2005 \mathrm{~b}$ ), there is no significant difference in the amount of springback even when using these three simple models, despite the fact that the predictions of a reverse shear test would be very different (see Fig. 7 in next section). This result is due to the low thickness/die radius ratio, combined with a significant friction force. Indeed, when a uniform stretching is superposed on pure bending, the stress gradient in the thickness is reduced and for a given amount of stretch, all stress values in a section will have the same sign. In this case, there will be no stress reversal during draw-bending and the only useful part of the hardening model would be its monotonic aspect. This hypothesis is further investigated in the next section.

Several numerical parameters are known to influence the prediction of springback. Li et al. (2002) have investigated in detail the impact of the number of through-thickness integration points, the difference between solid and shell elements etc. Here, the analysis is restricted to plane strain two-dimensional solid elements and to a fixed number of integration points through the thickness (four layers of elements through the thickness, i.e. eight integration points). Nevertheless, three different formulations of such elements, available in Abaqus/Standard, have lead to somewhat different springback profiles. The reduced integration element overestimates springback since its stiffness is too low, as compared to fully integrated elements. This FE phenomenon is due to the so-called hourglass modes or zero-energy modes which require an efficient stabilization technique. On the other hand, fully integrated linear elements are known to be too stiff, especially when loaded in bending, due to shear, and sometimes volume, locking. It is for these reasons that the linear plane strain element CPE4I of Abaqus (2003) is preferred in this work. This element provides selective reduced integration to prevent volume locking for almost incompressible (e.g. plastic) behavior, as well as incompatible modes additional degrees of freedom to prevent shear locking (Simo and Armero, 1992). These elements are particularly designed for an accurate description of bending strains. Moreover, the aspect ratio of the elements has been kept equal to one for all the simulations in order to preserve an optimal accuracy. This also allowed for a satisfactory number of elements along the radii of the punch and the die. In the worst case, the turning angle did not exceed $5.5^{\circ}$. Up to 5400 elements have been required to ensure these conditions for all the simulations given in the next section.

Nevertheless, it is important to note that the impact of the finite element formulation can be as important as the hardening law. For applications where greater accuracy is required, the development of advanced constitutive models may also require the development of accurate finite elements (see e.g. Yoon et al., 1999).

In conclusion, there is significant influence of the blank holding force, FE formulation and other numerical factors (Haddag et al., 2005) on the springback for this geometry. However, this geometry is not very sensitive to the hardening model. This makes such geometries not suitable to compare the two material models under study. Consequently, a different test geometry has been used in the following section for this purpose, since it exhibits "sharper" dimensions. 


\subsection{2. "Sharp" test geometry}

To highlight the influence of the hardening model on springback, a second strip drawing test geometry has been considered (Till and Raab, 2005). Indeed, the radii of the die and punch are smaller, thus inducing a stronger strain-path change over the die radius by bending/unbending with stretching during the drawing sequence. Hence, both the transient hardening and springback predicted by the two hardening models should differ.

As stated in several works (e.g. Pourboghrat and Chu, 1995; Carden et al., 2002; Geng and Wagoner, 2002; Lee et al., 2005a, 2005b; Haddag et al., 2004, 2005), the bending/unbending (with or without stretching) of the sheet is the principal mode of deformation that affects springback. The unbending sequence can be considered as a reverse loading and therefore the reverse shear test can be used as a typical representation of this mode of deformation, in order to interpret the difference on springback predicted with the two hardening models. Fig. 6 shows the reverse shear curves, predicted by the two models, at two amounts of pre-strain (10 and 30\%) and for each material. It is noteworthy that these two particular pre-strain levels have been used for the identification of the constitutive parameters, as indicated in the appendix.

The predictions of the two models differ strongly under non-monotonic strain-path, while they coincide under monotonic loading. Thus, the predicted springback can be almost model-independent (as it has been the case for the "smooth" geometry and as reported in literature), if the sheet undergoes a stretch-dominated strain history. Nevertheless, if the strain history becomes bending-dominated, the stress distribution at the end of the forming simulation will strongly depend on the material model implying different results in terms of springback. As a general trend, one can conclude that for a wide range of strain following the stress reversal, the classical model overestimates the stresses with respect to the microstructural one; only for the mild steel, a different situation may be expected for small post-reversal strains.

Consequently, an overestimated springback should be expected with the classical model. The simpler, purely isotropic or kinematic hardening models, should provide the extreme springback values in most cases, as suggested by their rheological predictions in Fig. 7. The predictions of these simple models are only provided in the paper as they represent well known academic bounds, while isotropic and kinematic hardenings occur simultaneously in the two advanced models compared in the current work.

The second test geometry has been selected to investigate the validity of this intuitive development. Fig. 8 and 9 show the springback predicted with the different models and for each material, corresponding to this new geometry. A high and a low BHF are used for each material. In these test conditions, higher strain levels are reached during the simulations. More specifically, the maximum amount of pre-strain at the end of the bending sequence ranges from 0.13 to 0.32 (equivalent strain), depending on sheet thickness, constitutive model and holding force. On one hand, one can conclude that the range of pre-strains is covered reasonably well by the rheological tests used for the parameter identification. On the other hand, these strains are about three times larger than the pre-strain levels reached with the "smooth" geometry. 
For the mild steel (see Fig. 8), there is a significant difference in the amount of springback as predicted by the two combined hardening models; the classical model predicts a larger springback for each BHF. This result correlates well with the previous intuitive statements based on the analysis of the stress reversal behavior. One can reasonably conclude that for this material and geometry, the bending/unbending loading is the predominant effect compared to the stretch due to the applied BHF. The predictions of the isotropic and the kinematic hardening models confirm this conclusion.

For the high strength steel (Fig. 9), however, a larger springback is obtained with the microstructural model when the low BHF is applied. The interpretation of this result simply from the reverse loading analysis is not straightforward, since the classical model still overestimates the stress during the second loading path, as shown in Fig. 6b - thus the same tendency should be obtained as for the mild steel. A realistic interpretation should take into account the actual strain history and the resulting stress distributions in the part before springback. It is obvious from Table 4 that, since the thickness of the two materials is not the same, the strain levels and the corresponding stress distributions through the thickness will be different as compared to the mild steel simulation. Thus, simple and general trends and interpretations cannot be easily deduced for such applications and the accurate numerical simulation of the process is required.

Again, when the stretching becomes important with respect to the bending, the difference in the amount of springback given by the two hardening models tends to vanish. This is achieved here by considerably increasing the blank holding force (see the high BHF in Fig. 9).

The computational efficiency of the numerical implementation has been analyzed for these springback simulations. For this purpose, all the simulations have been performed with the same number of time increments (11000 increments during the entire simulation). Table 6 gives the total number of equilibrium iterations of the finite element code required for each simulation and for each material model. This table clearly indicates the current implementation is as efficient as the built-in constitutive algorithms of Abaqus. Moreover, as shown in table 7, the computing time is not affected by the use of the Teodosiu model, in comparison to the Chaboche model or even to the Abaqus built-in models. This performance is due to two factors. First, in a static implicit FE code the CPU time is mainly related to the equilibrium resolution. Thus the constitutive algorithm has a reduced impact on the total computing time. Moreover, the nonlinear systems solved by the constitutive algorithms developed here have the same size for both material models. The solution time is therefore identical, although the accuracy and complexity of the two models are very different.

\section{Conclusion}

Two combined isotropic-kinematic hardening models, the classical Chaboche model and the microstructural Teodosiu-Hu model, are compared in this paper in order to show the impact of the transient hardening on springback. These models differ in their capability to reproduce the transient hardening phenomena which occur upon abrupt strain-path changes; the Teodosiu-Hu model is known to provide a better description of 
two-stage experimental tests. An implicit time integration algorithm has been developed and implemented in a FE code to simulate sheet metal forming processes. A springback analysis has been performed by means of a strip drawing benchmark test. The influence of the hardening model has been specifically highlighted. The following concluding remarks can be drawn:

- An implicit state update algorithm for a category of time independent, anisotropic, large strain elastoplasticity models has been developed and described in detail. The Chaboche model and the Teodosiu-Hu model are shown to fall into this category. This paper provides a framework for the numerical implementation of these models that can be readily applied to other similar models.

- The numerical implementation of this algorithm in the commercial FE code Abaqus/Standard is accurate and robust enough to simulate sheet forming operations, like the strip drawing tests analyzed in this paper. The computing time of the FE simulations does not depend on the selected hardening model, although the number of internal variables, as well as the complexity of the modeled behavior is very different between the two models.

- For the simulation of many forming operations, the choice of hardening law is less important than the uncertainty of other simulation parameters (mesh size, element type, number of integration points). The "smooth" geometry considered in this paper is an example of this type of operation.

- For some forming operations, however, the choice of hardening law is more important than these uncertainties. The "sharp" geometry considered in this paper falls into this category.

One should note that in these tests the predominant strain path is the strain reversal, due to the bending-unbending sequence; there is no orthogonal strain-path change. For such cases, other models (e.g. Chun et al. 2002, Yoshida et al. 2002) can also provide accurate simulations. For more complex industrial processes, e.g. involving multi-step operations, one can expect to further improve the simulation accuracy by using more physically-based models, such as the Teodosiu-Hu model.

\section{Acknowledgements}

This work has been carried out in a project funded by the European Commission, Research Fund for Coal and Steel (contract 7210-PA/371). The authors are grateful to Prof. Cristian Teodosiu from LPMTM (University Paris XIII) for fruitful discussions and guidance during this work.

\section{References}

ABAQUS, 2003. User's manual for version 6.4, Hibbitt, Karlson \& Sorensen Inc.

Abdel-Karim, M., Ohno, N., 2000. Kinematic hardening model suitable for ratcheting with steady-state. Int. J. Plasticity 16, 225-240. 
Abdel-Karim, M., 2005. Numerical integration method for kinematic hardening rules with partial activation of dynamic recovery term. Int. J. Plasticity 21, 1303-1321.

Alves, J.L., 2003. Simulação numérica do processo de estampagem de chapas metálicas: Modelação mecânica e métodos numéricos. Ph. D. Thesis, University of Minho, Portugal.

Armero, F., Pérez-Foguet, A., 2002. On the formulation of closest-point projection algorithms in elastoplasticity-part I: The variational structure. Int. J. Numer. Meth. Engng. 53, 297-329.

Armstrong, P.J., Frederick, C.O., 1966. A mathematical representation of the multiaxial Bauschinger effect. G.E.G.B. Report RD/B/N731.

Balan, T., Teodosiu, C., 1999. Constitutive algorithm for plasticity model of texture, strain-path induced anisotropy and rate-sensitivity: Interface between constitutive algorithm and FE code (Abaqus/ Standard). Internal Report, Paris 13 University.

Barlat, F., Ferreira Duarte, J.M., Gracio, J.J., Lopes, A.B., Rauch, E.F., 2003. Plastic flow for nonmonotonic loading conditions of an aluminum alloy sheet sample. Int. J. Plasticity 19, 1215.

Boger, R.K., Wagoner, R.H., Barlat, F., Lee, M.G., Chung, K., 2005. Continuous, large strain, tension/compression testing of sheet material. Int. J. Plasticity 21, 2319-2343.

Bouvier, S., Haddadi, H., Levée P., Teodosiu, C., 2006. Simple shear tests:

Experimental techniques and characterization of the plastic anisotropy of rolled sheets at large strains. J. Mater. Proc. Technol. 172, 96-103.

Bouvier, S., Teodosiu, C., Haddadi, H., Tabacaru, V., 2003. Anisotropic workhardening behavior of structural steels and aluminum alloys at large strains. Journal de Physique IV 105, 215-222.

Bouvier, S., Alves, J.L., Oliveira, M.C., Menezes, L.F., 2005. Modeling of anisotropic work-hardening behavior of metallic materials subjected to strain-path changes.

Computational Material Science 32 (3-4), 301-315.

Carden, W.D, Geng, L.M., Matlock, D.K., Wagoner, R.H., 2002. Measurement of springback. Int. J. of Mech. Sci. 44, 79-101.

Chaboche, J.L., 1986. Time independent constitutive theories for cyclic plasticity. Int. J. Plasticity 2 (2), 149.

Chaboche, J.L., 1989. Constitutive equations for cyclic plasticity and cyclic viscoplasticity. Int. J. Plasticity 5, 247-302.

Chaboche, J.L. 1991. On some modifications of kinematic hardening to improve the description of ratchetting effects. Int. J. Plasticity 7, 661-678. 
Chaboche, J.L., Cailletaud, G., 1996. Integration methods for complex plastic constitutive equations. Computer Methods in Applied Mechanics and Engineering 133 (1-2), 125-155.

Choi, Y., Han, C.-S., Lee, J.K., Wagoner, R.H., 2006. Modeling multi-axial deformation of planar anisotropic elasto-plastic materials, part I: Theory. Int. J. Plasticity 22, 1745-1764.

Choi, Y., Han, C.-S., Lee, J.K., Wagoner, R.H., 2006. Modeling multi-axial deformation of planar anisotropic elasto-plastic materials, part II: Applications. Int. J. Plasticity 22, 1765-1783.

Chu, C.-C., 1991. The effect of restraining force on springback. Int. J. Solids Struct. 27, 1035-1046.

Chun, B.K., Jinn, J.T., Lee, J.K., 2002a. Modeling the Bauschinger effect for sheet metals, part I: theory. Int. J. Plasticity 18, 571-595.

Chun, B.K., Jinn, J.T., Lee, J.K., 2002b. Modeling the Bauschinger effect for sheet metals, part II: applications. Int. J. Plasticity 18, 597-616.

Chung, K., Lee, M.-G., Kim, D., Kim, C., Wenner, M.L., Barlat, F., 2005. Springback evaluation of automotive sheets based on isotropic-kinematic hardening laws and nonquadratic anisotropic yield functions. Part I: theory and formulation, Int. J. Plasticity 21, 861-882.

De Montleau, P., Duchêne, L., Bouvier, S., Flores, P., Habraken, A.M., 2004. Deep drawing process with different elastoplastic laws. In: Esaform Conference Proceedings, Trondheim, 163-166.

Dongjuan, Z., Zhenshan, C., Xueyu, R., Yuqiang, L., 2006. Sheet springback prediction based on non-linear combined hardening rule and Barlat89's yielding function. Comp. Mat. Sci., in press.

Duffett, G., Weyler, R., Cruz, C., 2002. Material hardening model sensitivity in springback predictions. In: Conference Proceedings of Numisheet2002, 85-90.

Firat, M., 2006. U-channel forming analysis with an emphasis on springback deformation. Materials \& Design, in press.

Freed, A.D., Walker K.P., 1992. Exponential integration algorithms for first-order ODES with application to viscoplasticity, NASA TM 105.587, ASME Summer Conf. on Mechanics and Materials. Recent Advances on Damage Mechanics and Plasticity, Tempe.

Gau, J.T., Kinzel, G.L., 2001. A new model for springback prediction in which the Bauschinger effect is considered. Int. J. Mech. Sci. 43, 1813-1832. 
Genevois, P., 1992. Etude expérimentale et modélisation du comportement plastique anisotrope de tôles d'acier en grandes déformations. Institut National Polytechnique de Grenoble, France. PhD Thesis.

Geng, L., 2000. Application of plastic anisotropy and non-isotropic hardening to springback prediction. $\mathrm{PhD}$ Thesis. The Ohio State University, Columbus, $\mathrm{OH}$.

Geng, L., Shen, Y., Wagoner, R.H., 2002. Anisotropic hardening equations derived from reverse-bend testing. Int. J. Plasticity 18 (5-6), 743-767.

Geng, L., Wagoner, R.H., 2002. Role of plastic anisotropy and its evolution on springback. Int. J. Mech. Sci. 44, 123-148.

Haddadi, H., Bouvier, S., Levée, P., 2001. Identification of a microstructural model for steels subjected to large tensile and/or simple shear deformations. Journal de Physique IV 11, 329-337.

Haddadi, H., Tabacaru, V., Teodosiu, C., Maier, C., Bouvier, S., 2003. Tensile/Shear Tests and Identification of Elastoplastic Models for DP600, DP500 and DC06 Steels, Final report, LPMTM, University Paris 13, 54 pages.

Haddadi, H., Bouvier, S., Banu, M., Maier, C., Teodosiu, C., 2006. Towards an accurate description of the anisotropic behaviour of sheet metals under large plastic deformations: Modelling, numerical analysis and identification. Int. J. Plasticity 22, 2226-2271.

Haddag, B., Balan, T., Abed-Meraim, F., 2004. Finite element prediction of strain-path induced anisotropy in deep drawing. In: Esaform Conference Proceedings, Trondheim, 491-494.

Haddag, B., Balan, T., Abed-Meraim, F., 2005. Springback simulation: Impact of some advanced constitutive models and numerical parameters. In: Numisheet'05 Conference, Detroit, 286-291.

Hartmann, S., Haupt, P., 1993. Stress computation and consistent tangent operator using non-linear kinematic hardening models. Int. J. Num. Methods Engnrg. 36, 3801-3814.

He, N., Wagoner, R.H., 1996. Spring-back simulation in sheet metal forming. In: Conference Proceedings of Numisheet'96, 308-315.

Hill, R., 1950. The Mathematical Theory of Plasticity. Oxford University Press, Oxford.

Hoferlin, E., Li., S., Van Bael, A., Van Houtte, P., 2001. Texture- and microstructureinduced anisotropy: micro-macro modeling, implementation. In: Mori, K. (Ed.), Simulation of Materials Processing: Theory, Methods and Applications, Proc. of Inter. Conf. Numiform 2001. Swets \& Zeiltinger, Lisse, 209-214. 
Hughes, T.J.R., 1984. Numerical implementation of constitutive models: rateindependent deviatoric plasticity. In: Theoretical foundation for large-scale computations for nonlinear material behavior, Netherlands: Martinus Nij Publishers, 2957.

Keavey, M.A., 2001. A canonical form return mapping algorithm for rate independent plasticity. Int. J. Numer. Methods Engng. 53, 1491-1510.

Khan, A., Huang, S.H., 1995. Continuum Theory of Plasticity. John Wiley \& Sons, New York.

Khoei, A.R., Jamali, N., 2005. On the implementation of a multi-surface kinematic hardening plasticity and its applications. Int. J. Plasticity 21, 1741-1770.

Kuwabara, T., Morita, Y., Miyashita, Y., Takahashi, S., 1995. Elastic-plastic behavior of sheet metal subjected to in-plane reverse loading. In: Proceedings of Plasticity'95, Dynamic Plasticity and Structural Behavior. Gordon and Breach.

Kuwabara, T., Takahashi, S., Ito, K., 1996. Springback analysis of sheet metal subjected to bending-unbending under tension Part II (experimental verification). Advanced Technology of Plasticity. In: Altan T., editor. Proceedings of the $5^{\text {th }}$ ICTP, vol. II. Columbus, Ohio, 747-50.

Lee, M.-G., Kim, D., Kim, C., Wenner, M.L., Wagoner, R.H., Chung, K., 2005a. Springback evaluation of automotive sheets based on isotropic-kinematic hardening laws and non-quadratic anisotropic yield functions. Part II: Characterization of material properties. Int. J. Plasticity 21, 883-914.

Lee, M.-G., Kim, D., Kim, C., Wenner, M.L., Chung, K., 2005b. Springback evaluation of automotive sheets based on isotropic-kinematic hardening laws and non-quadratic anisotropic yield functions. Part III: Applications. Int. J. Plasticity 21, 915-953.

Lemaitre, J., Chaboche, J.L., 1990. Mechanics of solid materials. Cambridge University Press.

Li, K.P., Carden, W.P., Wagoner, R.H., 2002. Simulation of springback. Int. J. Mech. Sci. 44, 103-122.

Mattiasson, K., Strange, A., Thilderkvist, P., Samuelsson, A., 1995. Spring-back in sheet metal forming. In: Conference Proceedings of Numiform'95, pp. 115-124.

Miyauchi, K., 1984a. A proposal of a planar simple shear test in sheet metals. In: Scientific Papers of the Institute of Physical and Chemical Research, Vol. 78 (3), pp. $27-40$.

Miyauchi, K., 1984b. Bauschinger effect in planar shear deformation of sheet metals. Adv. Technol. Plasticity 1, 523. 
3DS Benchmarks, 2002. Results of the experimental benchmark tests, "Growth Programme, Research Project 3DS, Digital Die Design System, Contract G1RDCT2000-00104, IMS199900005".

NUMISHEET'93 Benchmark Problem, 1993. In: Makinouchi, A., Nakamachi, E., Onate, E., Wagoner, R.H. (Eds.), Proc. 2nd Int. Conf. on Numerical Simulation of 3D Sheet Metal Forming Processes - Verification of Simulation with Experiment, Isehara, Japan.

Ohno, N., Kachi, Y., 1986. A constitutive model of cyclic plasticity for nonlinear hardening materials. ASME, J. Appl. Mech. 53, 395-404.

Ohno, N., Wang, J.D., 1993a. Kinematic hardening rules with critical state of dynamic recovery: part I-formulation and basic features for ratcheting behavior. Int. J. Plasticity 9, 373-390.

Ohno, N., Wang, J.D., 1993b. Kinematic hardening rules with critical state of dynamic recovery: part II-application to experimental of ratcheting behavior. Int. J. Plasticity 9, 391-403.

Oliviera, M.C., Alves, J.L., Menezes, L.F., 2003. One step springback strategies in metal forming. In: Proc. of the $7^{\text {th }}$ Int. Conf. on Comp. Plasticity, COMPLAS, Barcelona.

Ortiz, M., Popov, E.P., 1985. Accuracy and stability of integration algorithms for elastoplastic constitutive relations. Int. J. Numer. Methods Engrg. 21, 1561-1576.

Pérez-Foguet, A., Armero, F., 2002. On the formulation of closest-point projection algorithms in elastoplasticity-part II: Globally convergent schemes. Int. J. Numer. Meth. Engng. 53, 331-374.

Pourboghrat, F., Chu E., 1995. Springback in plane strain stretch/draw sheet forming. Int. J. Mech. Sci. 36 (3), 327-41.

Prager, W., 1956. A new method of analyzing stresses and strains in work-hardening plastic solids. ASME, J. App. Mech. Trans. 78, 493.

Rauch, E.F., 1998. Plastic anisotropy of sheet metals determined by simple shear tests. Mater. Sci. Eng. A241, 179-183.

Sabourin, F., Morestin, F., Brunet, M., 2002. Effect of non-linear kinematic hardening on spring-back analysis. In: Conference Proceedings of Numisheet2002, pp. 79-89.

Simo, J.C., Armero, F., 1992. Geometrically nonlinear enhanced strain mixed methods and the method of incompatible modes. Int. J. Num. Methods Engnrg. 33, 1413-1449.

Simo, J., Taylor, R., 1985. Consistent tangent operators for rate-independent elastoplasticity. Computer Methods in Applied Mechanics and Engineering 48, 101-118. 
Stoughton, T.B., Yoon, J.-W., 2004. A pressure-sensitive yield criterion under a nonassociated flow rule for sheet metal forming. Int. J. Plasticity 20, 705-731.

Teodosiu, C., Hu, Z., 1995. Evolution of the intragranular microstructure at moderate and large strains: Modeling and computational significance. In: Simulation of Materials Processing: Theory, Methods and Applications. Numiform'95 Proceedings, 173-182.

Teodosiu, C. (editor), 1997. Large Plastic Deformation of crystaline aggregates. CISM courses and lectures - No. 376, ISBN: 3-211-82909.

Teodosiu, C., Hu, Z., 1998. Microstructure in the continuum modeling of plastic anisotropy. In: 19th Riso International Symposium on Materials Science Proceedings, Roskilde, 149-168.

Teodosiu, C., 2003. On the implementation of the dislocation-based microstructural model. Private Communication.

Till, E., Raab, A.E., 2005. Modeling effects in springback simulation. In: $24^{\text {th }}$ IDDRG Conference. Besançon.

Yoon, J.W., Yang, D.Y., Chung, K., 1999. Elasto-plastic finite element method based on incremental deformation theory and continuum based shell elements for planar anisotropic sheet materials. Comput. Methods Appl. Mech. Engrg., 174, 23-56.

Yoshida, F., Uemori, T., Fujiwara, K., 2002a. Elastic-plastic behavior of steel sheets under in-plane cyclic tension-compression at large strain. Int. J. Plasticity 18, 633-659.

Yoshida, F., Uemori, T., 2002b. A model of large-strain cyclic plasticity describing the Bauschinger effect and work hardening stagnation. Int. J. Plasticity 18, 661-686.

Ziegler, H., 1959. A modification of Prager's hardening rule. Quart. Appl. Math. 17, 5565 . 


\section{Figure captions}

Fig 1. Simulation of a tensile test with the classical model; validation of the numerical implementation.

Fig. 2. Rheological predictions with the microstructural model. a) Mild steel and b) Dual phase steel. UT: uniaxial tension; SS: simple shear; BS: Bauschinger shear at 10, 20 and $30 \%$ of pre-strain; OR: uniaxial tension up to $10 \%$ of pre-strain followed by simple shear in the same direction.

Fig. 3. Rheological predictions with the classical model. a) Mild steel and b) Dual phase steel. UT: uniaxial tension; SS: simple shear; BS: Bauschinger shear at 10, 20 and 30\% of pre-strain; OR: uniaxial tension up to $10 \%$ of pre-strain followed by simple shear in the same direction.

Fig. 4. The strip drawing test geometry.

Fig. 5. "Smooth" test geometry: shape of the strip after springback. Influence of a) the blank holding force (BHF), b) the hardening model and c) the formulation of the finite element.

Fig. 6. Comparison between the two hardening models in the reverse shear test at 10 and $30 \%$ amount of pre-strain: a) Mild steel and b) Dual phase steel.

Fig. 7. Predictions of the simple shear test, after $10 \%$ of pre-strain in shear in the opposite direction, with the purely isotropic hardening, the purely kinematic hardening, the classical Chaboche model and the microstructural (Teodosiu) model. a) Mild steel and b) DP steel.

Fig. 8. "Sharp" test geometry: shape of the strip after springback. Influence of the hardening model and of the blank holding force (BHF) for the mild steel a) Low blank holder force $(\mathrm{BHF}=24 \mathrm{kN})$; b) High blank holder force $(\mathrm{BHF}=72 \mathrm{kN})$.

Fig. 9. "Sharp" test geometry: shape of the strip after springback. Influence of the hardening model and of the blank holding force (BHF) for the Dual phase steel. a) Low blank holder force $(\mathrm{BHF}=84 \mathrm{kN})$; b) High blank holder force $(\mathrm{BHF}=324 \mathrm{kN})$. 


\section{Tables}

Table 1. Return mapping algorithm over one increment in the co-rotational frame

Table 2. Initial anisotropy: Hill'48 parameters for the two steels

Table 3. Hardening parameters of the two steels

Table 4. Strip test geometries

Table 5. Hardening parameters of the two steels - simplified models

Table 6. Total number of equilibrium iterations for the simulation of strip-drawing and springback with the sharp geometry

Table 7. Total CPU time (in hours) for the simulation of strip-drawing and springback with the sharp geometry 


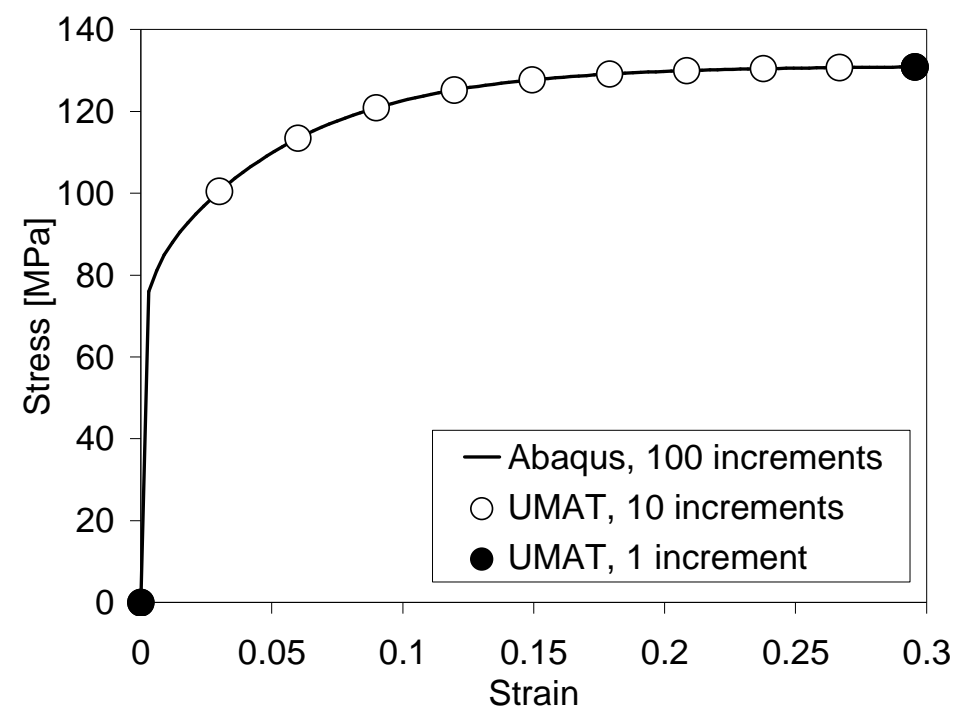

Fig 1. 

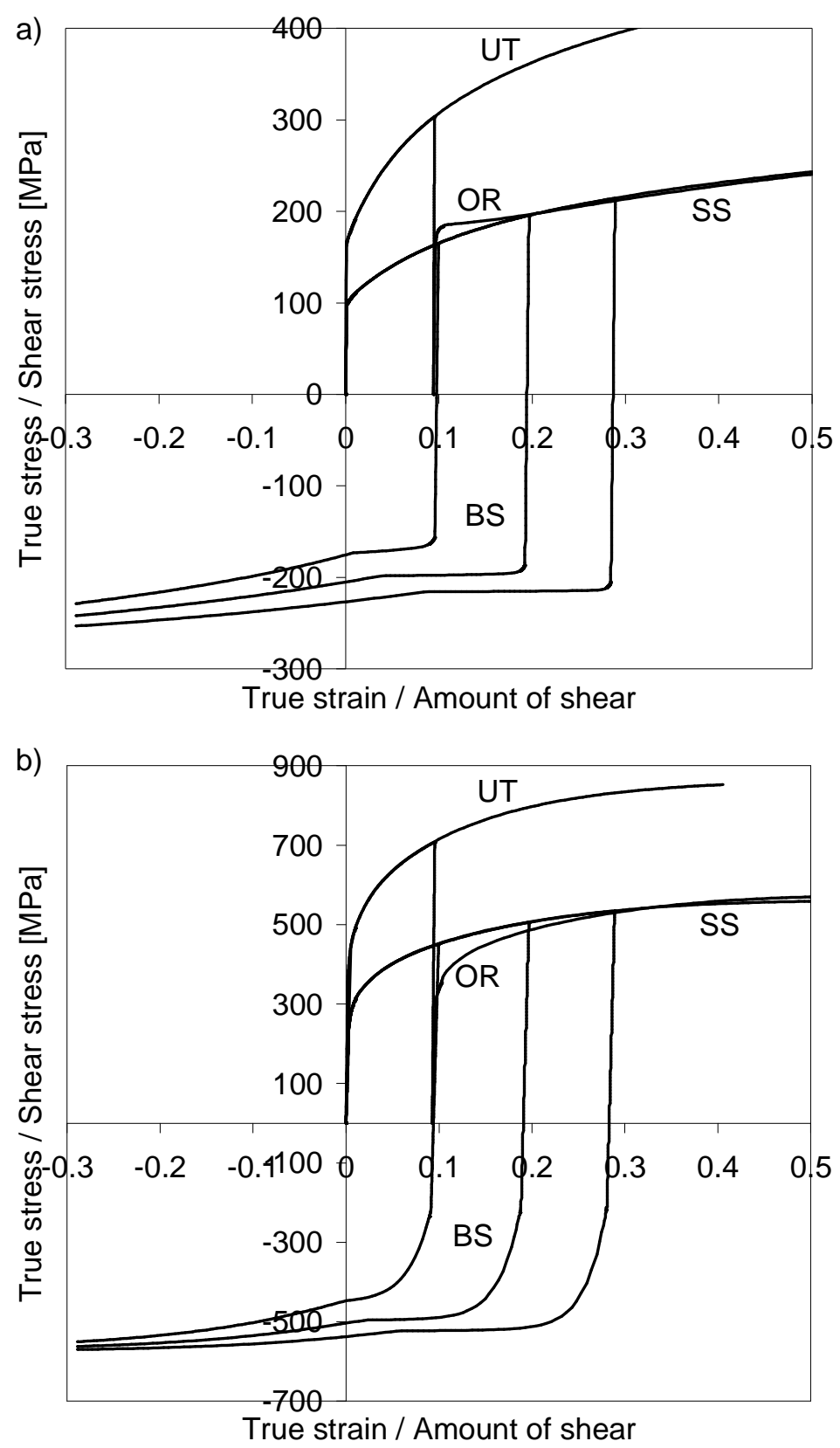

Fig. 2. 

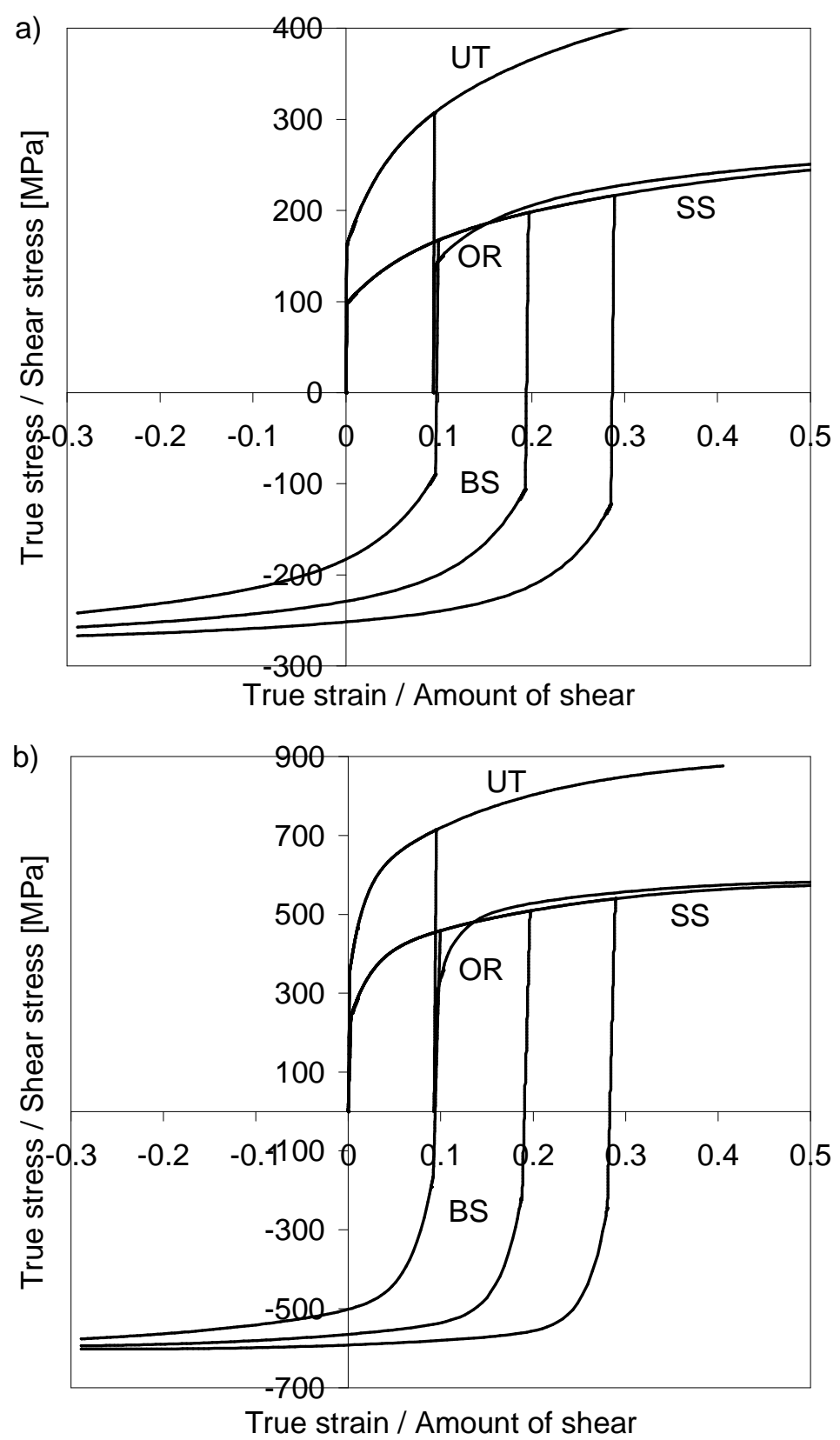

Fig. 3. 


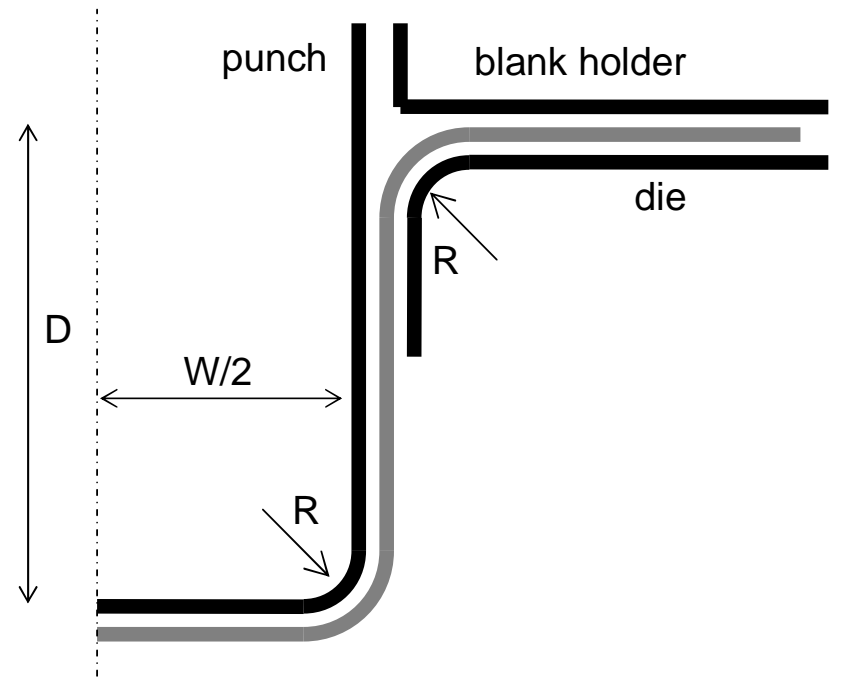

Fig. 4. 

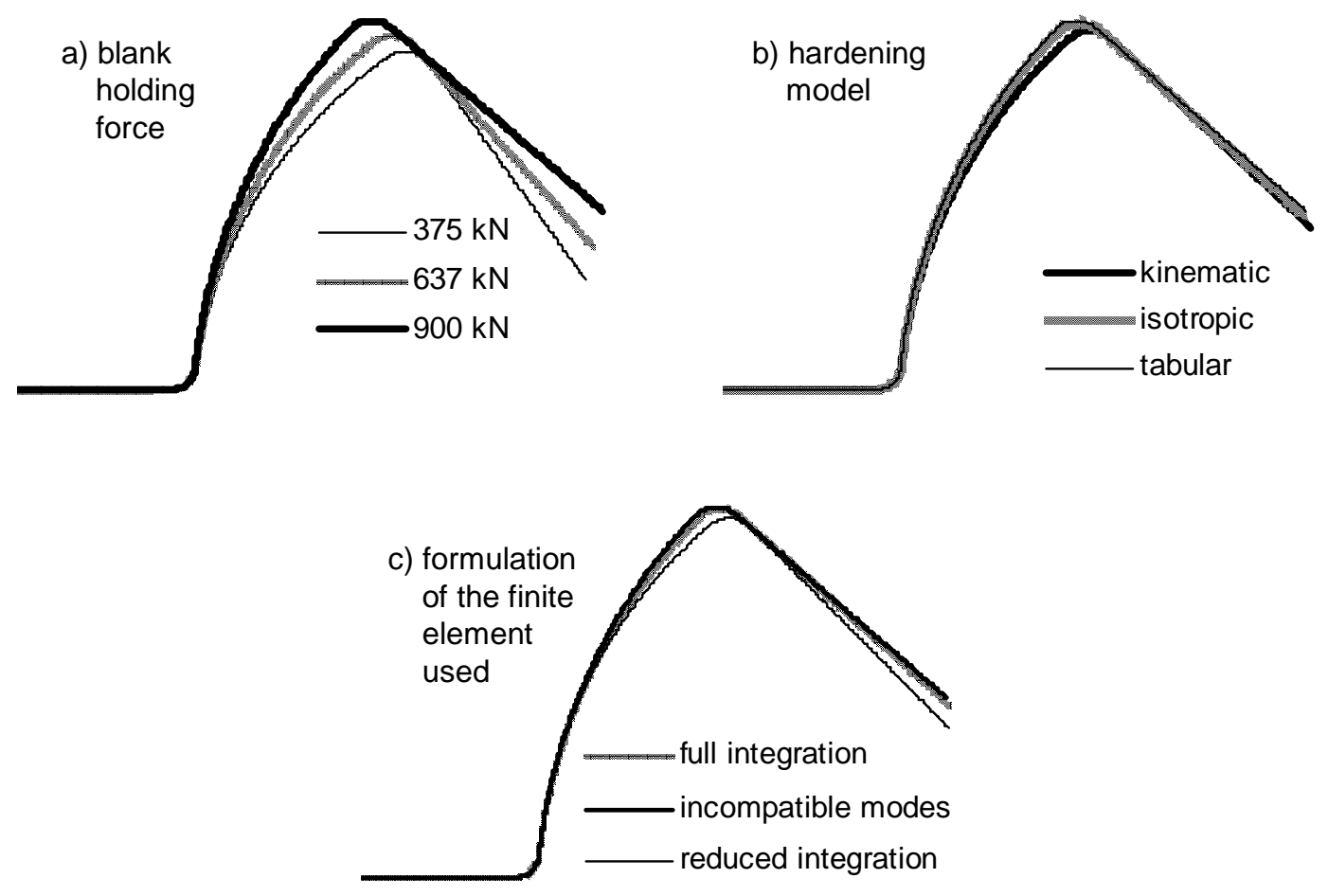

Fig. 5. 

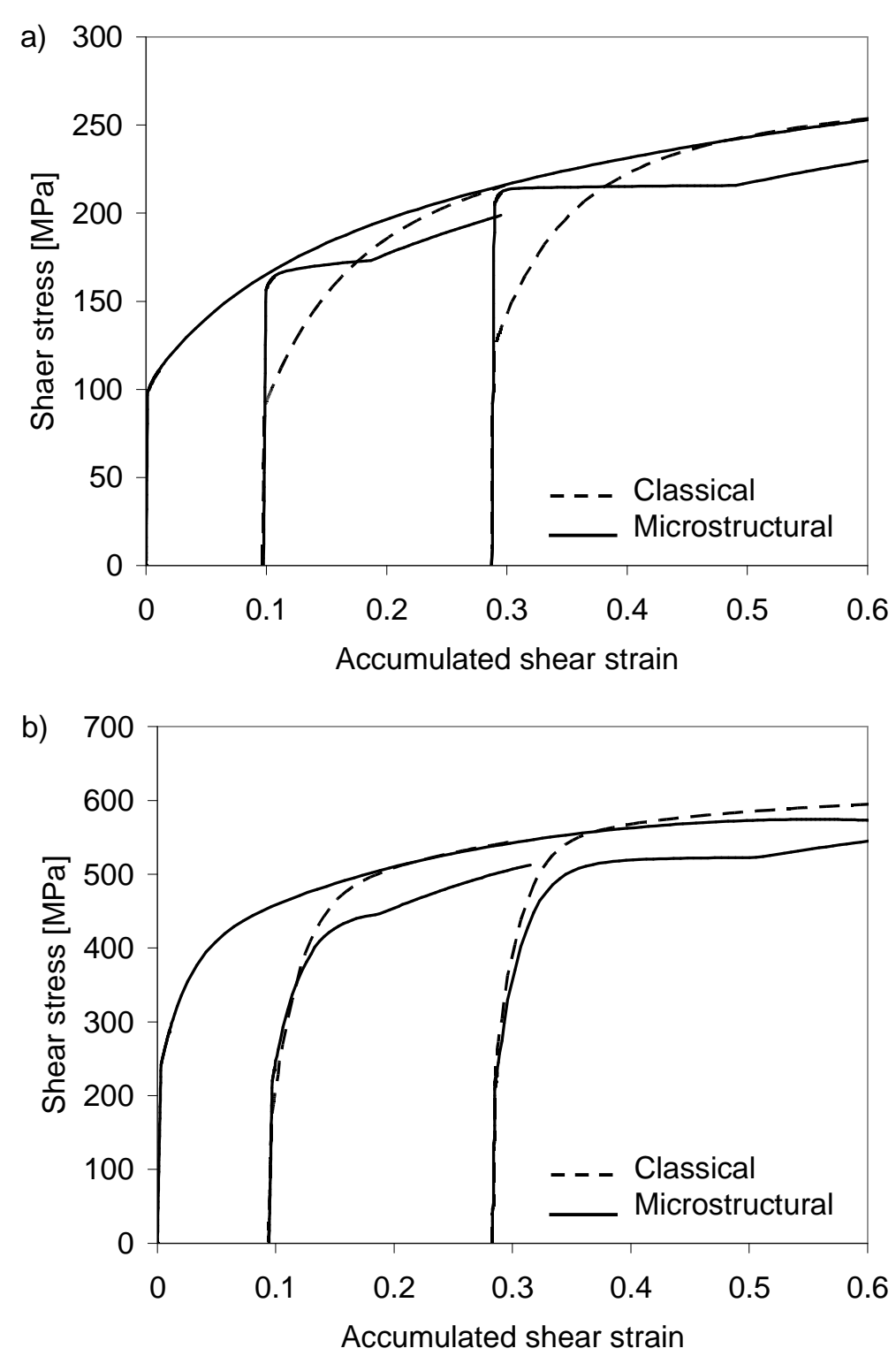

Fig. 6. 

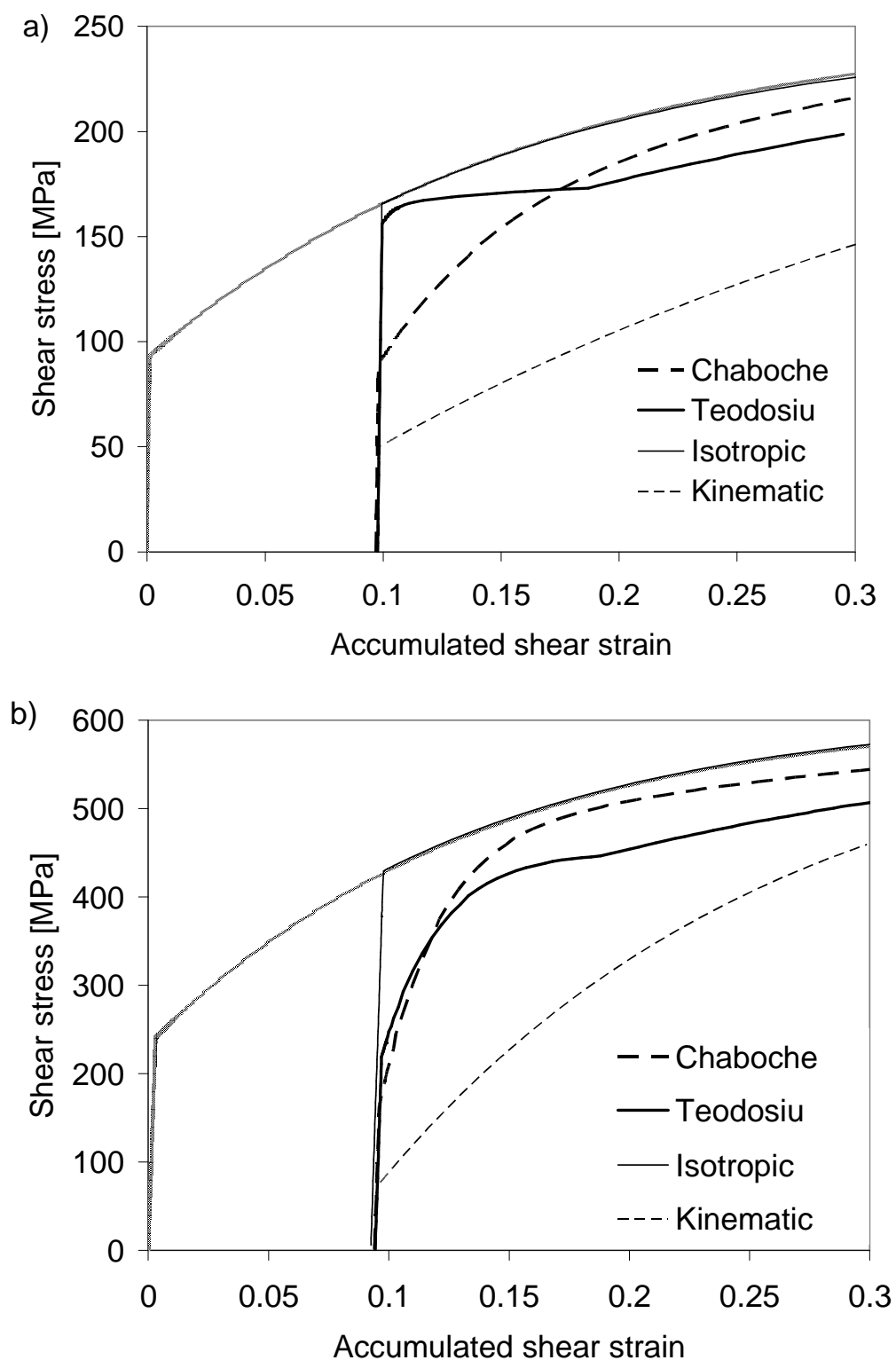

Fig. 7. 
a)

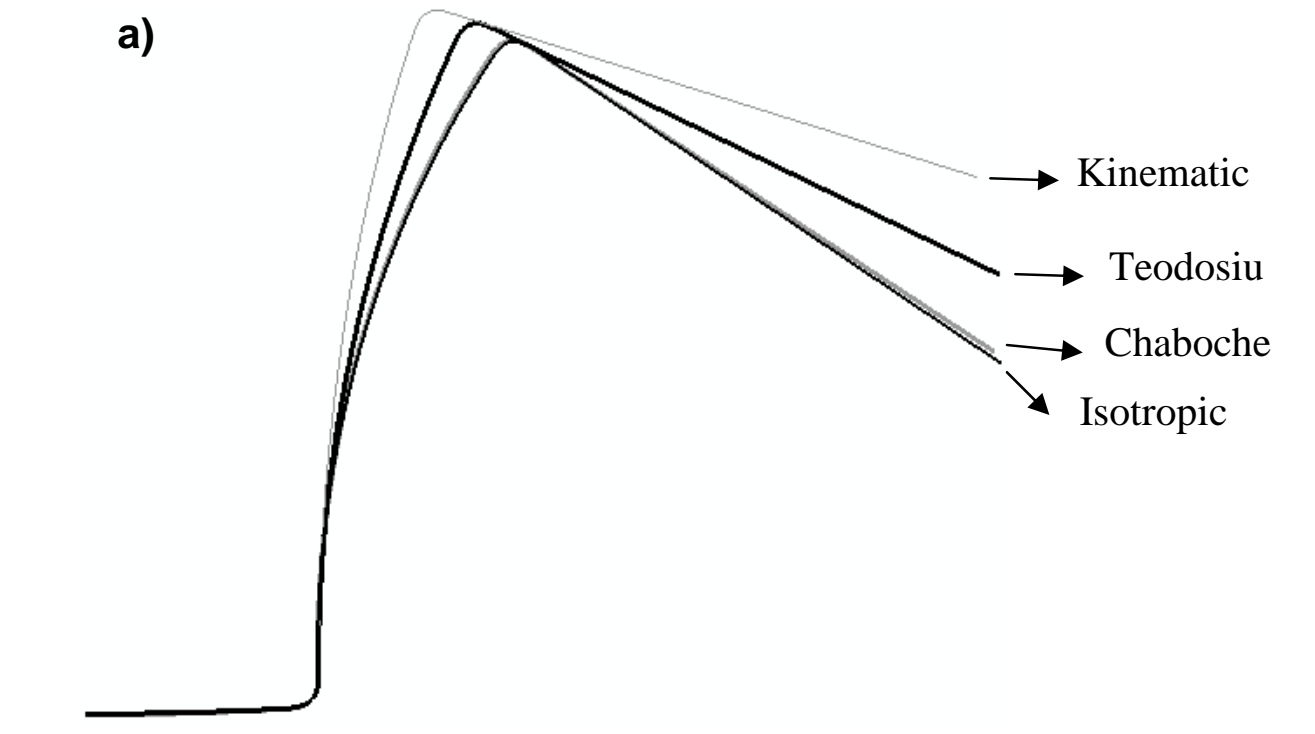

b)

Kinematic

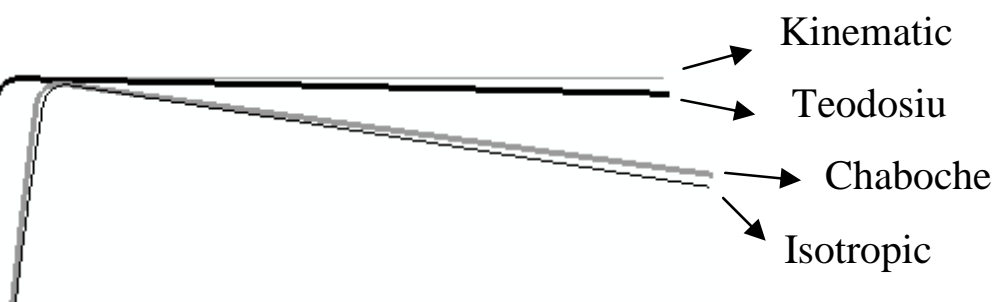

Fig. 8. 
a)



b)

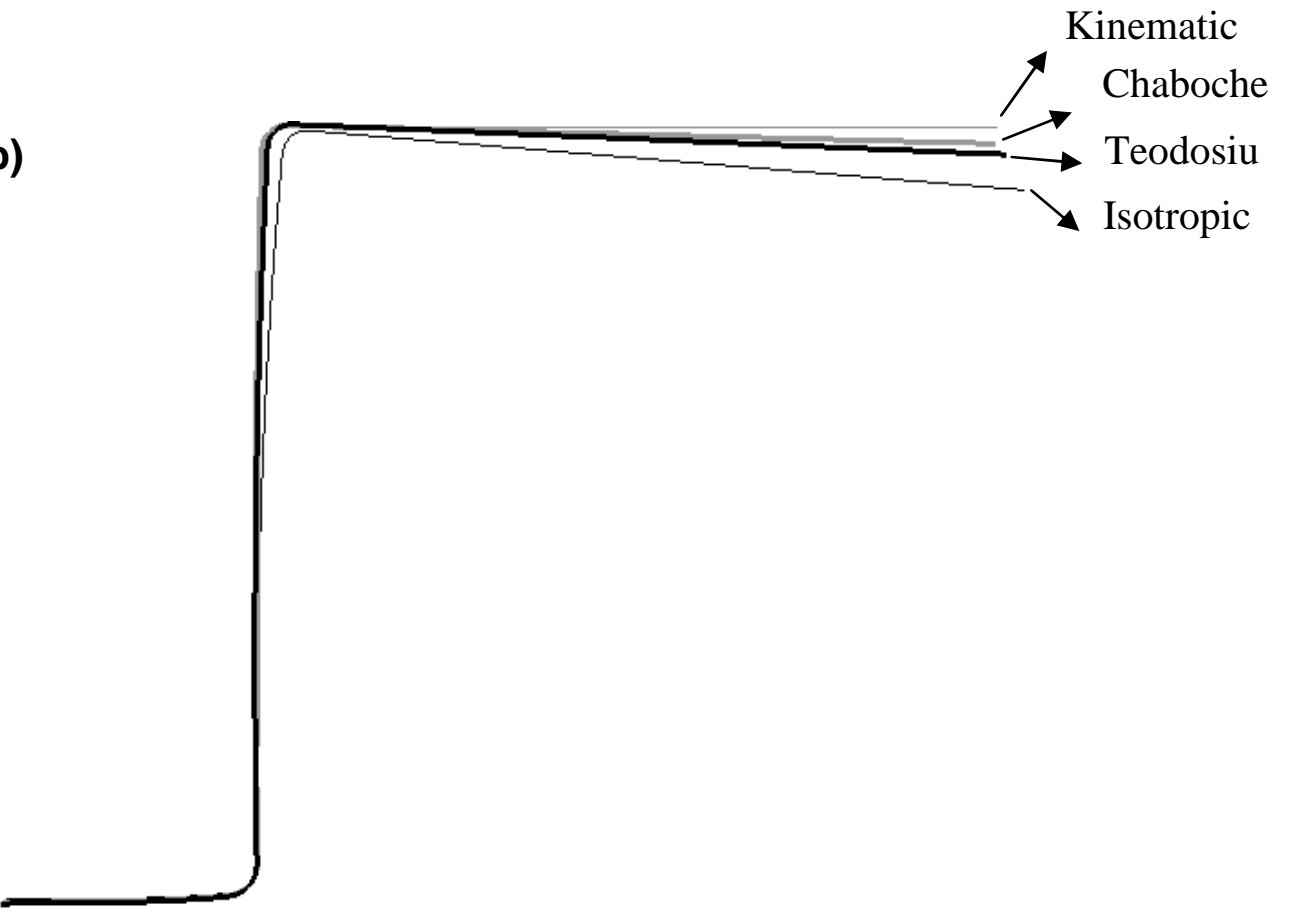

Fig. 9. 
Table 1

Return mapping algorithm over one increment in the co-rotational frame

1. Input data: strain increment, stress and internal state variables: $\Delta \boldsymbol{\varepsilon}, \boldsymbol{\sigma}_{n}$ and $\mathbf{y}_{n}$

2. Elastic prediction: $\boldsymbol{\sigma}_{n+1}^{t r y}=\boldsymbol{\sigma}_{n}+\mathbf{C}: \Delta \boldsymbol{\varepsilon}$

3. Check the yield criterion: $F_{n+1}^{t r y}=F\left(\boldsymbol{\sigma}_{n+1}^{t r y}, \mathbf{y}_{n}\right)<0$ ?

4. If elastic behavior then:

State update: $\boldsymbol{\sigma}_{n+1}=\boldsymbol{\sigma}_{n+1}^{t r y}$ and $\mathbf{y}_{n+1}=\mathbf{y}_{n} \rightarrow$ go to 8 .

Otherwise (i.e. elastoplastic behavior), continue.

5. Initialize: $\mathbf{T}_{n+1}=\boldsymbol{\sigma}_{n+1}^{\prime t r y}-\mathbf{X}_{n}$ and $\Delta \lambda=0$

6. Update $\mathbf{T}_{n+1}$ and $\Delta \lambda$ by solving Eq. (29) by Newton-Raphson.

7. State update:

$\mathbf{y}_{n+1}$ with Eq. (28)

$\boldsymbol{\sigma}_{n+1}$ with Eqs. (30) and (31)

8. Compute the consistent tangent modulus $\mathbf{C}^{\text {cons }}$ with Eq. (42).

9. Return $\boldsymbol{\sigma}_{n+1}$ and $\mathbf{C}^{\text {cons }}$ to check the equilibrium state. 
Table 2

Initial anisotropy: Hill'48 parameters for the two steels

\begin{tabular}{lcc}
\hline Material & Mild steel & Dual phase steel \\
Thickness $(\mathrm{mm})$ & 0.68 & 1.2 \\
\hline $\mathrm{F}$ & 0.234 & 0.428 \\
$\mathrm{G}$ & 0.339 & 0.562 \\
$\mathrm{H}$ & 0.662 & 0.438 \\
$\mathrm{~N}$ & 1.35 & 1.09 \\
$\mathrm{~L}, \mathrm{M}$ & 1.5 & 1.5 \\
\hline
\end{tabular}


Table 3

Hardening parameters of the two steels

\begin{tabular}{lccccc}
\hline & \multicolumn{3}{c}{ Mild steel } & \multicolumn{2}{c}{ Dual phase steel } \\
\hline & Classical & \multicolumn{2}{c}{ Microstructural } & Classical & Microstructural \\
\hline$Y_{0}(\mathrm{MPa})$ & & 161.7 & & 356.1 & \\
$R_{\text {sat }}(\mathrm{MPa})$ & 225.5 & 75.12 & 331 & 77.02 \\
$C_{R}$ & 4.14 & 23.29 & 5.88 & 558.9 \\
$X_{s a t}(\mathrm{MPa})$ & 78.26 & - & 220.4 & - \\
$C_{X}$ & 28.9 & 361.9 & 70.93 & 65.1 \\
$X_{0}(\mathrm{MPa})$ & & 7.3 & & 120.4 \\
$S_{s a t}(\mathrm{MPa})$ & & 233.3 & & 313.3 \\
$C_{S D}$ & & 3.75 & & 8.67 \\
$C_{S L}$ & & 1.097 & & 0 \\
$C_{p}$ & & 2.42 & & 1.53 \\
$n_{L}$ & & 0 & & 0 \\
$n_{p}$ & & 974 & & 700 \\
$f$ & & 1 & & 0.49 \\
$r$ & & 0.86 & & 0 \\
\hline
\end{tabular}


Table 4

Strip test geometries

\begin{tabular}{lcc}
\hline Dimensions & Test 1 "smooth" & Test 2 "sharp" \\
\hline$L / W$ & 4.4 & 6.4 \\
$D / W$ & 0.9 & 1.7 \\
$T / R$ & 0.2 & 0.23 and 0.4 \\
\hline
\end{tabular}


Table 5

Hardening parameters of the two steels - simplified models

\begin{tabular}{lccccc}
\hline & \multicolumn{2}{c}{ Mild steel } & \multicolumn{2}{c}{ Dual phase steel } \\
\hline & $\begin{array}{c}\text { Purely } \\
\text { isotropic }\end{array}$ & $\begin{array}{c}\text { Purely } \\
\text { kinematic }\end{array}$ & $\begin{array}{c}\text { Purely } \\
\text { isotropic }\end{array}$ & $\begin{array}{c}\text { Purely } \\
\text { kinematic }\end{array}$ \\
\hline$Y_{0}(\mathrm{MPa})$ & & 161.7 & & & 356.1 \\
$R_{\text {sat }}(\mathrm{MPa})$ & 303.75 & - & 551.40 & - \\
$C_{R}$ & 5.1 & - & 9.3 & - \\
$X_{\text {sat }} \quad(\mathrm{MPa})$ & - & 303.75 & - & 551.40 \\
$C_{X}$ & - & 5.1 & - & 9.3 \\
\hline
\end{tabular}


Table 6

Total number of equilibrium iterations for the simulation of strip-drawing and springback with the sharp geometry

\begin{tabular}{lcccc}
\hline & \multicolumn{2}{c}{ Mild steel } & \multicolumn{2}{c}{ Dual phase steel } \\
\hline BHF $(\mathrm{kN})$ & 24 & 72 & 84 & 324 \\
\hline Isotropic (Abaqus) & 23605 & 24657 & 20852 & 23725 \\
Kinematic (Abaqus) & 24546 & 25442 & 22851 & 24343 \\
Chaboche (Abaqus) & 24640 & 21868 & 22419 & 27799 \\
Chaboche (UMAT) & 24552 & 21905 & 22319 & 27466 \\
Teodosiu (UMAT) & 22120 & 22729 & 21944 & 23222 \\
\hline
\end{tabular}


Table 7

Total CPU time (in hours) for the simulation of strip-drawing and springback with the sharp geometry

\begin{tabular}{lcccc}
\hline & \multicolumn{2}{c}{ Mild steel } & \multicolumn{2}{c}{ Dual phase steel } \\
\hline BHF (kN) & 24 & 72 & 84 & 324 \\
\hline Isotropic (Abaqus) & 57.18 & 61.23 & 35.86 & 39.72 \\
Kinematic (Abaqus) & 60.81 & 62.28 & 38.41 & 40.42 \\
Chaboche (Abaqus) & 60.69 & 55.2 & 38.18 & 45.03 \\
Chaboche (UMAT) & 61.62 & 57.12 & 38.59 & 45.57 \\
Teodosiu (UMAT) & 57.66 & 58.35 & 38.61 & 40.01 \\
\hline
\end{tabular}

